\title{
Psychosomatische Störungen
}

\section{Therapeutische Verfahren bei psycho- somatischen Störungen im HNO-Bereich}

\section{E. Decot}

\section{Inhaltsverzeichnis}

$1 \quad$ Einleitung und Definitionen ... 273

2 Psychosomatische Diagnostik ... 273

2.1 Welche Möglichkeiten haben wir als HNO-Ärzte, um eine psychosomatische Diagnose zu stellen und eine entsprechende Therapie einzuleiten? ...273

2.2 Fragebögen...274

3 HNO-Erkrankungen mit psychosomatischer Komorbidität... 274

3.1 Erkrankungen der Ohren ... 274

3.1.1 Tinnitus ... 274

3.1.2 Hyperakusis und Phonophobie ...276

3.1.3 Hörsturz...276

3.1.4 Psychogene Hörstörung...276

3.1.5 Schwerhörigkeit und Taubheit...276

3.1.6 Morbus Menière...277

3.1.7 Schwindel...277

3.2 Erkrankungen der oberen Atemwege ... 277

3.2.1 Akute und rezidivierende Infektionen/akute Rhinitis ... 277

3.2.2 Chronische und allergische Rhinitis...277

3.2.3 Klinisches Öko-Syndrom (Sick-building-Syndrom) ... 278

3.3 Erkrankungen des Pharynx...278

3.3.1 Glossodynie...278

3.3.2 Globus pharyngeus ...279

3.3.3 Dysphagie und Phagophobie ... 279
3.4 Erkrankungen des Larynx...280

3.4.1 Psychogene Dysphonie und psychogene Aphonie ... 280

3.4.2 Laryngeale Dysfunktionen: Laryngospasmus und Laryngismus...280

3.5 Bruxismus und temporo-mandibuläre Dysfunktion ...280

3.6 Dysmorphophobie... 281

3.7 Maligne Erkrankungen des HNO-Gebietes ... 281

4 Übersicht therapeutischer Verfahren ... 282

4.1 Entspannungsverfahren...282

4.1.1 Autogenes Training...282

4.1.2 Progressive Muskelrelaxation ... 282

4.1.3 Biofeedback ...282

4.1.4 Yoga, Qi Gong, Tai Chi ...282

4.2 Hypnose...282

4.3 Gespräche im Rahmen der Psychosomatischen Grundversorgung ...282

4.4 Verhaltenstherapie...283

4.5 Tiefenpsychologisch fundierte Gesprächstherapie ...283

4.6 Psychopharmaka...283

5 Stellenwert der stationären psychosomatischen Behandlung ... 283

$6 \quad$ Prognose psychosomatischer Störungen und Ausblick in die Zukunft... 283

Literatur (Hinweis: erscheint nur in der Online-Ausgabe

Institutsangaben

Praxis für Hals-Nasen-Ohren-Heilkunde, Allergologie und Psychotherapie

Korrespondenzadresse

Dr. med. Elke Decot · Praxis für Hals-Nasen-Ohren-Heilkunde, Allergologie und Psychotherapie

Forsthausstraße 27·63303 Dreieich ·E-mail: DecotElke@aol.com

Bibliografie

Laryngo-Rhino-Otol 2005; 84 Supplement 1: 272-284 @ Georg Thieme Verlag KG Stuttgart · New York .

ISSN 0935-8943 · DOI 10.1055/s-2005-861125 


\section{Zusammenfassung}

Psychosomatische Störungen in der Hals-Nasen-Ohren-Heilkunde, wie zum Beispiel Tinnitus, Hörsturz, Schwindelerkrankungen, Globusgefühle, Dysphagien, Stimmstörungen und viele mehr, treten häufig auf. Sie sind meist multifaktoriell bedingt, wobei das bio-psycho-soziale Modell eine wichtige Rolle spielt. Der Erstkontakt mit dem psychosomatisch kranken Patienten und das Erheben der Erstanamnese ist bereits ein wichtiger Schritt zur psychosomatisch orientierten Therapie. Es wird ein Überblick über die wichtigsten HNO-Erkrankungen mit psychosomatischer Komorbidität und die wissenschaftlich evaluierten psychosomatischen Behandlungsmöglichkeiten gegeben. Des Weiteren werden wichtige psychosomatische Behandlungsmethoden vom Entspannungsverfahren bis zur analytischen Gesprächstherapie vorgestellt. Zum Schluss werden die Kriterien für eine ambulante wie auch für eine stationäre Behandlung diskutiert und die Vorteile einer psychosomatisch orientierten Therapie sowohl für den Patienten als auch für den Arzt dargestellt.

\section{Schlüsselwörter}

Psychosomatische Erkrankung · Hals-Nasen-Ohren-Erkrankung • Psychosomatische Therapie · Psychotherapie

1

Einleitung und Definitionen

\section{Was bedeutet „psychosomatische Störung“?}

Jeder Mensch unterliegt den Wechselwirkungen körperlicher und seelischer Prozesse.

Psychosomatische Medizin ist die Krankheitslehre, die psychischen Prozessen bei der Entstehung körperlicher Leiden eine wesentliche Bedeutung beimisst.

Psychosomatische Erkrankungen sind oft multifaktoriell bedingt, wobei somatogene und psychogene Einflüsse eine Rolle spielen und sowohl krankheitsverursachend als auch krankheitserhaltend wirksam sein können [1].

Als Organmediziner sind wir meist geneigt, den somatischen Aspekten einer Erkrankung den Vorzug in der Diagnostik und Behandlung unter Missachtung der Bedeutung psychischer und sozialer Faktoren zu geben. Wenn unsere Maßnahmen nicht den gewünschten Erfolg zeigen, sind wir oft genauso frustriert wie unsere Patienten. Die ärztliche Frustration spiegelt sich in Aussagen wie der folgenden wider: „Damit müssen Sie leben, da kann man nichts machen.“

Die daraus resultierende Unzufriedenheit im Beruf und BurnOut-Syndrome sind auch bei Ärzten an der Tagesordnung. Eine Studie der Abteilung für Psychosomatik und Psychotherapie der Universität Gießen ergab, dass die Hälfte der befragten Ärzte schätzten, dass ihr Lebensstil ihre Gesundheit beeinträchtigt bzw. stark beeinträchtigt. Mindestens 20 Prozent der Ärzte leiden an einem Burn-Out-Syndrom [2].
Die folgenden Ausführungen sollen dazu dienen, für die psychosomatischen Störungen zu sensibilisieren, eine Übersicht über die wichtigsten Erkrankungsbilder im HNO-Gebiet zu geben, kurz die psychosomatisch orientierte Anamnese und Diagnostik aufzuzeigen und entsprechende Therapiekonzepte zu erläutern.

\section{$2 \quad$ Psychosomatische Diagnostik}

Ein psychosomatischer Zusammenhang mit einer organischen Störung kann nicht alleine aus dem Fehlen eines adäquaten organischen Befundes hergeleitet werden. „Wir haben bei der Untersuchung nichts gefunden, also müssen Sie etwas Psychisches haben“, ist eine für den Patienten falsche und verletzende Feststellung, die impliziert, dass er nicht richtig krank sei und er sich seine Erkrankung nur einbilde oder simuliere.

$\mathrm{Zu}$ fordern ist in jedem Fall eine positive Diagnostik unter Berücksichtigung der soziobiografischen Anamnese und der aktuellen Lebenssituation [3], das bedeutet den Nachweis einer psychischen Störung wie Angst oder Depression oder den Nachweis eines psychischen Zusammenhanges wie etwa der Beginn eines Ohrgeräusches zeitgleich mit einem heftigen Streit.

\subsection{Welche Möglichkeiten haben wir als HNO-Ärzte, um eine psychosomatische Diagnose zu stellen und eine entsprechende Therapie einzuleiten?}

Unser wichtigstes ärztliches Instrument ist neben unserem Fachwissen die Fähigkeit, einem Patienten einfühlsam und vorurteilsfrei zuzuhören und bereits im Erstgespräch eine vertrauensvolle Arzt-Patienten-Beziehung aufzubauen. Dazu gehört auch, die „richtigen“ Fragen zu stellen. Wir versuchen damit, die körperlichen Symptome des Patienten in einen verstehbaren $\mathrm{Zu}$ sammenhang mit seiner Lebensgeschichte und seiner emotionalen Situation zu bringen.

Im Folgenden wird ein Untersuchungsablauf dargestellt, der sowohl psychische als auch organische Aspekte in angemessener Weise berücksichtigt.

Folgende Fragen sind dabei essenziell:

\section{Frage 1}

\section{„Welche Beschwerden haben Sie?“}

Die Schilderung soll in den eigenen Worten des Patienten erfolgen - „ich leide an einem permanenten Klingeln im Ohr“ und nicht „ich habe Tinnitus“. Der Arzt achtet hierbei darauf, wie der Patient sich selber darstellt und welche Emotionen im Hintergrund zu spüren sind („ich bin verunsichert“, „ich leide“, „ich bin genervt").

\section{Frage 2}

„Wann war das Symptom zum ersten Mal da, was hat die Beschwerden ausgelöst, welche Ereignisse traten zeitgleich damit auf?"

Des Weiteren wird nach Verschlechterungen oder Besserungen gefragt. Hier gilt es für den Arzt oft hartnäckig zu sein, denn Patienten sagen zunächst meist „das weiß ich nicht mehr“ oder „es ist immer gleich schlimm“. 
Der HNO-Arzt kann zu diesem Zeitpunkt mit seiner körperlichen Untersuchung beginnen und dabei das Gespräch mit dem Patienten weiter fortführen. Bitte beachten Sie, dass auch bei Patienten, die bereits auf den ersten Blick psychisch krank erscheinen, auf eine organische Abklärung der Beschwerden nie verzichtet werden darf. Das Ergebnis der körperlichen Untersuchung sollte dem Patienten in verständlicher und respektvoller Form („die Hörprüfung hat eine normale Hörschwelle ergeben“ und nicht „Sie haben nichts“) mitgeteilt werden.

\section{Frage 3}

\section{„Hat sich in dieser Zeit in Ihrem Leben etwas Wesentliches verändert? Gab es schlimme Erlebnisse?"}

Der nächste wichtige Punkt ist die Frage nach der individuellen Lebenssituation des Patienten zum Zeitpunkt des Beschwerdebeginns. Das bio-psycho-soziale Modell von Uexküll [4] sollte hierbei bedacht werden: Krankmachende Faktoren können im biologischen, psychischen und sozialen Bereich liegen und Gesundheit bedeutet Wohlbefinden in all diesen Bereichen.

Der Arzt achtet hier auf mögliche Verbindungen zwischen Erkrankung und lebensgeschichtlichen Krisen (Jobverlust, Scheidung, Tod einer Bezugsperson etc.).

\section{Frage 4}

\section{„Haben Sie schon früher Probleme mit den Ohren/der Nase gehabt?“}

Welche Krankheitserfahrung hat der Patient im betroffenen Organsystem, hatte er zum Beispiel schon immer Probleme mit den Ohren oder hat auch die Mutter schon immer unter Kopfschmerzen gelitten? Hier wird die lebens- und familiengeschichtliche Einordnung aus der Sicht des Patienten deutlich.

\section{Frage 5}

„Was haben Sie bisher unternommen?“

Was hat der Patient bisher getan, um wieder gesund zu werden, welche Bewältigungsstrategien (Coping) hat er bereits entwickelt? Wie erfolgreich war er damit? Bei wie vielen Kollegen wurden bereits Behandlungsversuche unternommen? Diese Frage gibt Aufschluss darüber, was bereits versucht worden ist, und welche Möglichkeiten der Therapien noch bestehen.

\section{Frage 6 \\ „Wie stellen Sie sich die Behandlung vor?“}

Mit dieser Frage wird die Bereitschaft zur aktiven Mitarbeit thematisiert. Eine aggressive Antwort des Patienten wie etwa „Das müssen Sie doch wissen, Sie sind doch der Doktor!" signalisiert auch eine mangelnde Bereitschaft, aktiv mitzuarbeiten und Verantwortung für sich zu übernehmen. Einen Patienten gegen seinen Willen zu behandeln ist sinnlos, ihn beispielsweise ohne vorherige eigene Motivation zum Psychotherapeuten zu schicken meist ohne großen Erfolg. Ebenso ist es nicht ratsam, eine naturheilkundliche oder homöopathische Behandlung ohne Nachfragen, warum der Patient diese bevorzuge, mit „die taugen alle nichts" herabzuwürdigen.

Der zeitliche Aufwand einer psychosomatisch orientierten Anamnese ist bei einem geübten Untersucher nur unwesentlich länger als die „normale“ HNO-Anamnese. Die Berücksichtigung des bio-psycho-sozialen Modells bietet den unschätzbaren Vor- teil, dass sich der Patient mit seinen Beschwerden angenommen und ernst genommen fühlt und Vertrauen zum Arzt aufbauen kann. Des Weiteren ist diese Art der Anamnese schon ein Teil der Therapie: Sie fördert die Einsicht des Patienten in psychosomatische Zusammenhänge und reduziert seine Abwehr gegen diesbezügliche Therapievorschläge.

\section{$2.2 \quad$ Fragebögen}

Fragebögen sind hilfreich und können Zeit sparen, ersetzen aber nicht das ärztliche Gespräch. Vor dem Einsatz eines Fragebogens sollte der Untersucher eine klare Vorstellung darüber haben, was er erfahren möchte. Des Weiteren muss der Patient zur Mitarbeit bereit sein.

Der Tinnitusfragebogen nach Goebel und Hiller (TF) erfasst zum Beispiel die kognitive und emotionale Beeinträchtigung durch Tinnitus [5]. Das Beck'sche-Depressions-Inventar (BDI) gibt Hinweise auf den Schweregrad einer depressiven Symptomatik. Das Beck'sche Angstinventar (BAI) ermöglicht eine exakte Aussage über das Vorhandensein und den Schweregrad klinisch relevanter Angst. Das revidierte Freiburger Persönlichkeitsinventar (FPI-R) erfragt geschlechts- und altersspezifische Persönlichkeitsfaktoren wie Lebenszufriedenheit, soziale Orientierung, Leistungsorientierung, Aggressivität, Gesundheitssorgen, körperliche Beschwerden und mehr. Alle eben genannten Fragebögen sind validiert und zeitlich akzeptabel. Weitere Informationen zu Fragebögen gibt die Testzentrale des Hogrefe-Verlags in Göttingen.

\section{HNO-Erkrankungen mit psychosomatischer Komorbidität}

\subsection{Erkrankungen der Ohren 3.1.1 Tinnitus}

Tinnitus, das Hören von Geräuschen ohne äußere Schallquelle, ist so etwas wie eine typische Erscheinung der Zeit, in der wir leben. Das Symptom wird zur Krankheit, wenn der Patient beginnt, zu leiden. Die Therapie des Tinnitus war vor 50 Jahren nahezu ohne Bedeutung, heute im Medienzeitalter ist sie ein Massenphänomen und Millionengeschäft. Die Anzahl der angebotenen Therapieformen und therapeutischen Kombinationen [6,7], die Vielfalt der verwendeten Begriffe und der Mangel an vergleichenden Studien machen eine Beurteilung extrem schwierig. Hinzu kommt, dass gute Studien selten sind [8]. Es werden die verschiedensten Therapieelemente miteinander, teilweise auch mit medikamentösen Verfahren, kombiniert. Eine Vergleichbarkeit ist daher kaum möglich.

Die bisher populärste Form all dieser Kombinationsverfahren, die Tinnitus-Retraining-Therapie, die von Hazell und Jastreboff etabliert wurde, hat aufgezeigt, dass Counselling-Beratung ein wichtiges und erfolgreiches Therapieelement in der Behandlung des chronischen Tinnitus ist $[9,10]$. Es gibt mittlerweile diverse Varianten mit unterschiedlichen Erfolgsquoten [11].

Ich möchte den Begriff des Counsellings noch besonders erwähnen. Hierbei handelt es sich um eine krankheitsbezogene Beratung, bei der sich der HNO-Arzt die Zeit nimmt, dem Patienten organische und psychische Besonderheiten und Zusammenhän- 
ge seiner Symptome zu erklären; etwas was eigentlich eine Selbstverständlichkeit sein sollte. Es gibt Untersuchungen, die dies als den wesentlichen Bestandteil der Tinnitus-RetrainingTherapie ansehen [12].

Folgende therapeutische Einzelkomponenten werden in den meisten Therapien in unterschiedlicher Weise kombiniert: Autogenes Training, Progressive Muskelrelaxation nach Jacobson, Biofeedback, Hypnose, Tai Chi, Qi Gong, Yoga, Akupunktur, tiefenpsychologisch fundierte Gesprächstherapie, kognitive Verhaltenstherapie. Bei den meisten Patienten scheint es sinnvoll zu sein, mehrere Verfahren zu kombinieren, wobei verhaltenstherapeutische Methoden insgesamt häufiger verwandt werden als analytische [13].

Chronischer Tinnitus bei Erwachsenen, Kindern und Jugendlichen kann am besten als psychophysiologische Störung unter Berücksichtigung psychologischer und sozialer Aspekte erklärt werden. Die Beeinträchtigung der Lebensqualität kann erheblich sein und begleitend treten oft Angststörungen, Depressionen sowie Konzentrations- und Schlafstörungen auf [14]. Die Patienten fühlen sich sowohl auf der emotionalen als auch auf der kognitiven Ebene beeinträchtigt [15]. Interessant ist, dass bereits in frühen Stadien des Tinnitus interindividuelle Unterschiede bestehen, die auf eine hohe psychische Belastung, Depressivität sowie maladaptive Stressverarbeitung hindeuten [16] und somit eine frühzeitige psychosomatische Intervention sinnvoll erscheinen lassen.

Randomisierte und kontrollierte Studien weisen eine Wirksamkeit von kognitiver Verhaltenstherapie, Biofeedback und progressiver Muskelrelaxation nach [17-21]. Kognitive Verhaltenstherapie ist als stressreduzierende Maßnahme auch via Internet wirksam [22,23]. Alleiniges Counselling und Gruppentherapie nach Kröner-Herwig erwiesen sich als vergleichbar wirkungsvoll [24]. Der Aufmerksamkeitslenkung kommt in der Tinnitustherapie eine besondere Bedeutung zu, sie kann mit unterschiedlichen psychologischen Verfahren gefördert werden [25]. Entspannungsverfahren sind als Methode der Aufmerksamkeitslenkung auf positive Krankheitsbewältigungsaspekte grundsätzlich empfehlenswert.

Biofeedback erwies sich besonders dann als effektiv, wenn Muskelverspannungen und Stresssituationen im Vorfeld nachgewiesen wurden [26]. Die Zufriedenheit der Patienten war in der Regel hoch, bedingt durch eine verbesserte Fähigkeit, mit dem Ohrgeräusch umzugehen. Die Tinnitustagebücher ergaben aber nur geringe Effekte auf den Tinnitus selbst [18].

Selbsthypnose und aufmerksames Zuhören ärztlicherseits sind ebenfalls in der Lage, die Schwere des Tinnitus zu reduzieren [27]. In einer skandinavischen Studie berichteten 73 Prozent der Patienten ein Verschwinden des Geräusches während der Sitzungen im Vergleich zu 24 Prozent bei Behandlung mit einem akustischen Stimulus [28]. Ein Langzeiteffekt war nur in der Selbsthypnose-Gruppe nachweisbar.

Die Kombination von Verhaltenstherapie mit Entspannungsverfahren im Sinne des Erlernens von Coping-Strategien erbrachte eine signifikante Reduktion der Belästigung durch das Ohrge- räusch und eine Verbesserung der Stimmungslage über einen dreimonatigen Follow-up [29]. Weiterhin fanden sich positive Effekte hinsichtlich Schlafstörungen, Kopfschmerzen und Schwindelgefühlen. Bei dieser Studie gab es allerdings keine Kontrollgruppe. Andere Autoren bestätigen die Wirksamkeit dieser multimodalen Therapieformen [17,30].

In all diesen Studien wurden die Effekte der Therapiegruppen in der Regel mit einer Kontrollgruppe, die als Wartekontrolle geführt wurde, verglichen. Kritisch anzumerken ist, dass es keine einzige Studie gibt, die einen Rosenthaleffekt sicher ausschließt. Hierzu bedarf es einer Kontrollgruppe, die die gleiche ärztliche Zeit und Aufmerksamkeit erfährt wie die Verumgruppe, aber mit einer nachgewiesenermaßen wirkungslosen Therapie. Ein weiterer Kritikpunkt sind die zum Teil sehr kleinen Gruppen von nur wenigen Patienten.

Keine Wirksamkeit wurde für Akupunktur in Studien nachgewiesen. Ein einzelner Fallbericht beschrieb eine positive Wirkung von Tai Chi auf das Ohrgeräusch [31].

Ebenso war Yoga als alleinige Tinnitustherapie ohne nachweisbare Effekte [32].

Hypnose als Verfahren der Tiefenentspannung zeigte positive Effekte, erwies sich aber in der Kombination mit kognitiver Verhaltenstherapie und Stressmanagement breiter wirksam. Lediglich einige Fallbeschreibungen und kleinere Studien berichten über erfolgreiche Heilungen oder Besserungen durch Hypnose alleine [33-36]. Die Patienten kamen besser mit ihrem Ohrgeräusch zurecht, obwohl Lautstärke und Qualität unverändert waren [37].

Fazit für die Praxis: Wir empfehlen, bereits beim Erstgespräch den Patienten auf die Bedeutung psychischer Faktoren, entweder als Ursache oder als Folge des Tinnitus, hinzuweisen. Des Weiteren ist ein neurootologisches Counselling bei allen Hörstörungen sinnvoll - ein Patient, der die Zusammenhänge versteht, hat weniger Angst [38,39]. Beim dekompensierten Tinnitus sollten die soziale Unterstützung, soziale Stressbelastung sowie CopingStrategien als wichtige Prognosefaktoren beachtet werden [40]. Je mehr der Patient unter Tinnitus leidet, desto stärker profitiert er von einer integrierten psychosomatischen und otologischen Therapie [41]. Psychotherapeutische Gespräche in Kombination mit verschiedenen Entspannungsverfahren wie Progressive Muskelrelaxation, Biofeedback oder auch Hypnose sind wirkungsvoller als Entspannungsverfahren alleine. Psychologische Verfahren scheinen langfristig erfolgreicher zu sein als medikamentöse Behandlungen, die oft nur kurzfristige Effekte erzielen [8]. Die meisten Studien beziehen sich auf kognitive Verhaltenstherapie, weniger auf tiefenpsychologisch fundierte Gesprächstherapie. Beides sind Verfahren, die nur von ausgebildeten Psychotherapeuten durchgeführt werden können, also eine Überweisung seitens des HNO-Arztes erfordern.

Für den HNO-Arzt in Praxis und Klinik durchführbar und abrechenbar sind Gespräche im Rahmen der psychosomatischen Grundversorgung von mindestens 20 Minuten Dauer. Meiner Erfahrung nach gelingt es bei den meisten Patienten mit etwa fünf Gesprächen eine Stabilisierung und Besserung des Krankheits- 
bildes oder eine Motivation für eine weitere psychotherapeutische Therapie zu erzielen.

\subsubsection{Hyperakusis und Phonophobie}

Hyperakusis bedeutet eine Überempfindlichkeit über das gesamte Frequenzspektrum des Hörvermögens. Bei einer ängstlichen Persönlichkeit oder Angststörung kann es zu einer Phonophobie kommen, einer stark gesteigerten Empfindlichkeit gegenüber speziellen Geräuschen unabhängig von deren Lautstärke [38]. Hyperakusis kommt oft infolge eines Tinnitus vor. Wie beim Tinnitus sind die Schulung der Hörwahrnehmung und Aufmerksamkeitslenkung wichtige Therapiebausteine [42]. Die psychosomatische Therapie orientiert sich hier an der Angstkomponente, die im Fokus der Behandlung stehen sollte. Der Abbau des Vermeidungsverhaltens und die Neubewertung angstauslösender, akustischer Reize stehen dabei im Mittelpunkt der Therapieplanung. Weiterhin sind kognitiv verhaltenstherapeutische Ansätze geeignet. Expositionsbehandlung, positive Verstärkung und soziales Kompetenztraining haben sich in Einzelfallbeschreibungen als wirksam dargestellt. Ebenso wie beim Tinnitus ist der zusätzliche Einsatz von Entspannungsverfahren sinnvoll.

Fazit für die Praxis: Was für den Tinnitus gilt, trifft zum Teil auch für die Hyperakusis und die Phonophobie zu. Die Beratung des Patienten über physiologische und psychische Besonderheiten, wie in diesem Fall die Angstkomponente, ist von entscheidender Bedeutung. Entspannungsverfahren jeder Art wirken angstlösend und sind sinnvoll in die Behandlung zu integrieren.

\subsubsection{Hörsturz}

Beim Hörsturz hat der Patient einen plötzlichen und nachweisbaren Innenohrschaden erlitten. In verschiedenen Untersuchungen zeigte sich, dass in über 70 Prozent der untersuchten Fälle [43] der Hörsturz in akut psychisch belastenden Situationen auftrat. In der Regel konnte dabei die akute Belastung als Dekompensation eines chronischen Konfliktes aufgefasst werden. Hoher Leistungsanspruch, Pflichtbewusstsein, ausgeprägte Sensibilität und eine Unterdrückung aggressiver Impulse verbunden mit Schuldgefühlen sind oft charakteristische Eigenschaften der Patienten $[44,45]$. In einer Studie [46] wurde die psychische Struktur von Patienten mit Hörsturz untersucht. Patienten mit einer neurotischen Persönlichkeitsstruktur profitierten nicht von einer organischen Therapie.

Psychophysiologische Faktoren können ebenfalls rheologische Faktoren beeinflussen. Die Therapie des Hörsturzes sollte daher sowohl organisch orientierte Verfahren, wie in den Leitlinien der DGHNO empfohlen, als auch psychisch orientierte Verfahren beinhalten.

Eine Säule der Therapie bei diesen psychisch belasteten und angespannten Patienten sind logischerweise Entspannungsverfahren.

Prognostisch positiv wirkten sich tragfähige Beziehungsstrukturen, eine emotional stabile Persönlichkeit sowie die Reduktion von Stressfaktoren aus [47].
Fazit für die Praxis: Bereits beim Erstgespräch sollte nach akuten als auch chronischen Stressbelastungen gefragt werden. Die Patienten sind sich dieser oft bewusst und reagieren auf das ärztliche Interesse an ihrer Person meist positiv. Neben dem Angebot organischer Behandlungsverfahren sind Ermunterungen Entspannungsverfahren zu lernen äußerst sinnvoll. Wenn Autogenes Training oder Progressive Muskelrelaxation in der Praxis nicht möglich sind, können beispielsweise Kurse bei der Volkshochschule vorgeschlagen werden. Die in der letzten Zeit häufiger angebotenen und von vielen Patienten gerne ausgeübten fernöstlichen Übungen wie Tai Chi, Qi Gong und Yoga dienen ebenso der Entspannung. Der Patient sollte nur dann krankgeschrieben werden, wenn eine Krankschreibung seine Stressbelastung auch wirklich reduziert. Sollte der Patient zu Hause stark belastet sein oder Angst um seinen Arbeitsplatz haben, ist dies ärztlicherseits mit zu berücksichtigen.

\subsubsection{Psychogene Hörstörung}

Unter psychogener Hörstörung wird eine unbewusste, meist symmetrische, doppelseitige Schwerhörigkeit mittleren bis hohen Grades verstanden. Charakteristisch ist, dass die Störung nur bei den subjektiven Hörprüfungen angegeben wird und bei den objektiven Verfahren (OAE, BERA) nicht nachweisbar ist. Auch eine ungezwungene Unterhaltung oder Telefonieren ist ohne Probleme möglich [4]. Psychotherapeutisch gesehen handelt es sich meist um ein Konversionssymptom. Bei Kindern konnten Konfliktsituationen im schulischen und familiären Bereich beobachtet werden [48]. Es wird vermutet, dass es sich bei einem Teil der plötzlichen Hörstürze um psychogene Hörstörungen handelt, die nicht erkannt werden [49]. Randomisierte Diagnostik- und/ oder Therapiestudien hierzu gibt es nicht.

Fazit für die Praxis: Der Patient benötigt Zeit, um über seine Probleme, Ängste und Befürchtungen und nicht nur über seine Schwerhörigkeit zu sprechen. Es ist sinnlos, ihn unverblümt mit der Tatsache zu konfrontieren, dass er keinen organischen Hörschaden hat. Dagegen ist es essenziell für die Therapie, dass der Patient erkennt, welche Bedeutung die subjektiv empfundene Schwerhörigkeit in seinem Leben hat. Das Ziel ist dem Patienten zu ermöglichen, die Zusammenhänge zwischen der Schwerhörigkeit und seinen psychischen Problemen zu erkennen. Die Bewältigung der Konfliktsituation ist eine der wichtigsten Voraussetzungen zur Überwindung der psychischen Fehlhaltung [50]. Einige Autoren empfehlen auch gerade bei Kindern suggestive Behandlungsmethoden [51].

Bei Erwachsenen und bei kurzer Krankheitsdauer ist die Prognose im Allgemeinen gut. Bei Kindern sollte eine psychiatrische Erkrankung ausgeschlossen werden.

\subsubsection{Schwerhörigkeit und Taubheit}

Der hochgradige Hörverlust bis zur Taubheit, erworben oder angeboren, stellt für einen Menschen einen massiven Schicksalsschlag dar. Hier handelt es sich also um eine somatopsychische Situation. Darüber wie viele schwerhörige Patienten verbittert und depressiv soziale Kontakte abbrechen und vereinsamen gibt es bisher keine Statistiken. Aber jeder HNO- und Hausarzt kennt dieses Problem. Der Schwerhörige oder Gehörlose ist nicht nur ein normales Individuum minus Gehör, sondern er selbst und oft auch seine Mitmenschen zweifeln an seinen intellektuel- 
len Fähigkeiten und seiner sozialen Kompetenz. Dies hat oft aggressives Verhalten zur Folge. Schwerhörigkeit wird daher meist lange verheimlicht. Die mangelnde Akzeptanz Hörgeräte zu tragen ist das sichtbare Zeichen der Abwehr des Patienten. Bei schwerhörigen Kindern ist ebenso der psychische Zustand der Eltern zu berücksichtigen, die in vielen Fällen über eine deutlich erhöhte psychische Belastung auch über den Zeitpunkt der Diagnosestellung hinaus klagen.

Aus diesen Überlegungen resultiert der therapeutische Ansatz (Studien gibt es nicht).

Fazit für die Praxis: Empathie, das bedeutet mitfühlendes Zuhören und Hineinversetzen in die Lage des Patienten (Balint), sind nonverbale Kommunikationsmittel, die bei der Betreuung von Schwerhörigen und Tauben eine entscheidende Rolle spielen. Diese Patienten benötigen ärztliche Zeit, um ihre Nöte und Ängste auszudrücken und Aggressionen abzubauen.

\subsubsection{Morbus Menière}

Die klassischen Hauptsymptome des Morbus Menière sind attackenweise auftretender Drehschwindel mit Übelkeit, Tieftonhörverlust und Tinnitus. Die ohne Vorwarnung plötzlich auftretenden Anfälle lösen bei vielen Patienten Angst und phobische Reaktionen aus. Der „richtige“ psychosomatisch orientierte Umgang mit der ersten traumatisierenden Attacke ist von entscheidender Bedeutung für den weiteren Verlauf der Erkrankung [52]. Unumstritten ist die Tatsache, dass Stress als Begleitumstand oder Auslöser beim Morbus Menière eine Rolle spielt [53].

Fazit für die Praxis: Wichtig von Seiten des Arztes ist empathisches Verstehen für die vom Patienten als existenziell bedrohlich angesehene Situation, eine Aufklärung über das physiologische Geschehen beim Morbus Menière sowie über die möglichen organischen und psychologischen Therapieverfahren. Die Kombination von einem vestibulären Training und einem körperbetonten Entspannungsverfahren, wie zum Beispiel Progressiver Muskelrelaxation, hat sich unserer Erfahrung nach als hilfreich erwiesen.

\subsubsection{Schwindel}

Schwindel ist eine subjektive Empfindung einer Gleichgewichtsstörung in physischer und/oder psychischer Hinsicht [4].

Schwindel ist besonders in der Allgemeinmedizin ein häufiges Leitsymptom und oft die psychophysiologische Begleitreaktion auf Stress, Angst und Depression [54,55]. Ebenso können Angst und starke Unsicherheit Schwindelsymptome auslösen [56]. Eine einseitige, lediglich somatische Abklärung unter Missachtung psychosomatischer Faktoren birgt die Gefahr einer Chronifizierung.

Angsterkrankungen wie zum Beispiel Agoraphobie (Platzangst) gehen oft mit einem Gefühl des „Schwindelig-Seins“ einher. Diese Erkrankungen sind eine Domäne der Verhaltenstherapie. Eine akute Schwindelattacke ist ein kritisches Lebensereignis und als solches angstauslösend. Mangelnde Coping-Strategien und dysfunktionelle Kognitionen können zu einer Persistenz der Angst und damit der Schwindelsymptomatik führen [57].
Bei Kindern ist eine psychosomatische Erkrankung oft von dem Gefühl des „Schwindelig-Seins“ begleitet [58].

Kognitive Verhaltenstherapie in Kombination mit einem vestibulären Training hat sich bei der Rehabilitation als effektiv erwiesen, ohne dabei allerdings einen Einfluss auf Angst oder Depression zu haben [59].

Fazit für die Praxis: Die Abklärung psychischer Faktoren und eine gegebenenfalls interdisziplinäre Behandlung sollte schon zu Beginn einer Schwindelerkrankung erfolgen, um eine Chronifizierung zu vermeiden [60]. Eine entsprechende Psychoedukation des Patienten auch von Seiten des HNO-Arztes ist erforderlich, damit der Patient seine Situation realistisch einschätzen kann („mir ist schwindelig“ oder „ich habe Angst, dass mir schwindelig wird“) [61]. Vorangegangene Belastungssituationen und Verlusterlebnisse müssen exploriert und gegebenenfalls in einen für den Patienten verständlichen Zusammenhang mit seiner Schwindelsymptomatik gebracht werden, um zu vermeiden, dass er sich frustriert für einen Simulanten hält. Die Kombination eines vestibulären Trainings mit Entspannungsverfahren erscheint auch hier sinnvoll. Bei ausgeprägter Angststörung ist der Einsatz von Psychopharmaka zu überlegen [62].

\subsection{Erkrankungen der oberen Atemwege}

\subsubsection{Akute und rezidivierende Infektionen/akute Rhinitis}

Jeder von uns, sei er Patient oder Arzt, kennt das Phänomen, dass er schneller einen Infekt bekommt, wenn er unter Stressbelastung steht (Nachtdienst!). Stressbelastung kann zu einem erhöhten Auftreten von Atemwegsinfektionen führen. Erhöhter psycho-sozialer Stress reduziert die lokale Immunantwort gegen virale oder bakterielle Infektionen mit der Folge einer erhöhten Anfälligkeit gegenüber Erkältungskrankheiten [63].

Stressmanagement ist in der Lage, Krankheitstage zu reduzieren, und zwar unabhängig von der sIgA-Sekretion oder einer negativen Stimmungslage [64]. Entspannungstherapie bei Kindern mit positiven Suggestionen (Vermehrung der Immunglobuline) führte zu einer Erhöhung des sIgA- als auch des sIgA/Albumin-Quotienten als Parameter der lokalen Schleimhautimmunität [65].

Fazit für die Praxis: Therapiestudien gibt es nicht. In Anbetracht der Studienergebnisse zur Ätiologie von Infektionen der Atemwege erscheint es gerade bei rezidivierenden Beschwerden sinnvoll zu sein, eine erhöhte Stressbelastung zu erfragen und den Patienten auf diesbezügliche Zusammenhänge aufmerksam zu machen. Ein Plan für Stressmanagement und Entspannungsverfahren als Prophylaxe sind sinnvolle Maßnahmen.

\subsubsection{Chronische und allergische Rhinitis}

Die chronische Rhinitis ist ein häufiges Krankheitsbild. Die charakteristischen Symptome sind eine verstopfte Nase, Naselaufen und Niesreiz. Die Beschwerden sind meist in unterschiedlicher Ausprägung vorhanden und bestehen über Jahre. Durch die dauerhaften Beschwerden und die damit verbundene Beeinträchtigung kann es zu psychischen Störungen kommen (somato-psychische Genese). 
Untersuchungen zu psychosomatischen Aspekten sind selten, Therapiestudien nicht vorhanden. Umgangssprachliche Ausdrücke wie „die Nase von etwas voll haben“ beschreiben bildlich aber einen Zusammenhang von körperlichen Symptomen und der psychischen Verfassung.

Allergische und psychosomatische Reaktionen scheinen auf den ersten Blick keine Gemeinsamkeiten zu haben. Das eine beinhaltet ein klar definierbares immunologisches Geschehen, das andere beschreibt eine objektiv schwer erfassbare und im psychoimmunologischen Ablauf kaum zu beweisende Reaktion.

Psycho-immunologische Studien haben aufgezeigt, dass allergische Reaktionen durch hypnotische Suggestionen gehemmt werden können [66]. In experimentellen Studien konnte weiterhin belegt werden, dass die Histaminkonzentration im Plasma unter Stress ansteigt [67]. Allergien können durch Autosuggestion entstehen und starke Ängstlichkeit lässt die Haut sensibler auf potenzielle Allergene reagieren. Mehrere Studien aus Ungarn $[68,69]$ zeigen eine Korrelation von allergischen Beschwerden mit Depression und Angst. 74 Prozent der untersuchten Patienten mit Panikstörungen zeigten auch eine behandlungsbedürftige Typ-1-Allergie.

Auch beim Menschen konnte der Zusammenhang von Stress und erhöhter allergischer Reaktion belegt werden, allerdings sind diese Untersuchungen meist bei Asthma und Neurodermitis durchgeführt worden [70]. Während es bei Asthma und Neurodermitis bereits Therapiestudien und psychosomatisch orientierte Behandlungskonzepte $[71,72$ ] gibt, liegen zur allergischen Rhinitis bisher keine brauchbaren therapeutischen Konzepte oder Studien vor, so dass hierzu bisher nur spekuliert werden kann.

Fazit für die Praxis: Eine Allergie ist ein multifaktorielles Geschehen. Gerade bei perennialen Allergikern oder bei multiplen Sensibilisierungen spielen auch nach meinen Erfahrungen Angst und Depression als Komorbidität eine Rolle. Ob sie schon vor der Allergie bestanden oder im Rahmen einer chronischen, die Lebensqualität beeinträchtigenden Erkrankung aufgetreten sind, ist nicht zu beurteilen.

Therapeutisch ist es sinnvoll, den Patienten auf die Möglichkeit des Vorliegens einer zusätzlichen Angst und/oder Depression aufmerksam zu machen - „ich kann verstehen, dass diese ständigen Beschwerden Sie depressiv werden lassen oder Sie Angst haben, dass es immer schlimmer wird". Auch hier können suggestive Entspannungsverfahren je nach psychischer Ausgangslage des Patienten Erleichterung bringen. Des Weiteren ist wie bei allen chronischen Erkrankungen das Erlernen von Coping-Strategien sinnvoll. Bei Verdacht auf eine relevante Angststörung oder Depression ist die Überweisung zum Fachpsychotherapeuten angezeigt. Wenn sich der Patient vom Arzt ernst genommen fühlt und er das ärztliche Bemühen um Hilfe spürt, wird auch eine Überweisung zum Psychotherapeuten nicht mehr so problematisch sein.

\subsubsection{Klinisches Öko-Syndrom (Sick-building-Syndrom)}

Hierbei handelt es sich um Patienten, die nach einer vermeintlichen oder tatsächlich vorhandenen Belastung mit chemischen Substanzen eine massive Überempfindlichkeit gegenüber jed- wede Art von Chemikalien entwickelt haben. Eine organisch fassbare Schädigung ist zumeist nicht nachweisbar, des Weiteren auch keine diesbezüglichen Allergien. Die Beschwerden der Patienten sind oft massiv, die Lebensqualität erheblich eingeschränkt.

Bisher konnten bei den betroffenen Patienten von ärztlicher Seite aus nur psychosomatische und zum Teil auch neurotische Störungen festgestellt werden. Dies wird von den meisten Patienten jedoch vehement abgelehnt, so dass entsprechende therapeutische Versuche zum Scheitern verurteilt sind. Bisher gibt es keine brauchbaren Behandlungskonzepte von psychosomatischer oder psychiatrischer Seite [71].

Fazit für die Praxis: Die betroffenen Patienten sind oft sehr schwer zugänglich, insbesondere wenn von ärztlicher Seite die Kausalität bestimmter chemischer Substanzen für die Beschwerden infrage gestellt wird. Oft sind schon diverse Therapien auch aus dem alternativen Bereich versucht worden. Die Patienten sind mit ihrem hohen Leidensdruck „leichte Beute“ für unseriöse Anbieter.

Das wichtigste therapeutische Ziel ist hier, eine stabile Arzt-Patienten-Beziehung aufzubauen. Der Arzt soll Ansprechpartner bleiben, auch wenn er selber keine Möglichkeit einer Behandlung sieht, um den Patienten vor Schaden zu bewahren.

\subsection{Erkrankungen des Pharynx 3.3.1 Glossodynie}

Patienten mit Glossodynie oder Burning-Mouth-Syndrom gehören zu den „schwierigen“ Patienten. Betroffen sind meist Frauen mittleren Alters. Sie sind fokussiert auf ihre Organbeschwerden, sie jammern, sie leiden unter starken Schmerzen, sie fordern organbezogene Untersuchungen und Therapien und verweigern oft jede Einsicht in eine psychosomatische Störung. Die Diskrepanz zwischen dem Erleben des Patienten und den nicht objektivierbaren Läsionen im Mundschleimhautbereich ist erheblich. Das Arzt-Patienten-Verhältnis ist dementsprechend problematisch [73]. Die Patienten erleben sich selbst als psychisch normal. Die Ätiopathogenese ist zumeist unbekannt. Patienten, die unter Fibromyalgie leiden, geben auch gehäuft Schmerzen im Mundbereich an [74]. Behandlungsstudien mit größeren Fallzahlen gibt es nicht, meist handelt es sich um Einzelfallberichte. Wenn HNO-ärztlicherseits eine organische Ursache ausgeschlossen wurde, wird oft eine psychogene Genese angenommen. Depressionen und Angsterkrankungen werden meist als Komorbidität oder auch als Auslösefaktoren angegeben [75]. Andere Autoren sehen Persönlichkeitsveränderungen als entscheidender an als neurotische Symptome wie Depression [76]. Ebenso spielen Stressfaktoren bei vielen Patienten eine entscheidende Rolle.

Daher ist zumindest zu Beginn der Behandlung die Gabe von Antidepressiva gegebenenfalls auch Anxiolytika in Kombination mit Gesprächstherapie in Erwägung zu ziehen [77]. Es gibt einige Studien mit Empfehlungen für Verhaltenstherapie, aber evidence-based ist die Datenlage nicht. Biofeedback und Hypnose als Entspannungsverfahren zur Reduktion von Angstzuständen sowie kognitive Verhaltenstherapie, um maladaptive Gedankenmuster der Patienten zu verbessern, sind ebenfalls Therapiemöglichkeiten in ausgewählten Einzelfällen [78]. 
Fazit für die Praxis: Das Ziel der Behandlung ist der Aufbau einer stabilen Arzt-Patienten-Beziehung. Sie ist die Voraussetzung jeglicher Therapieversuche und die einzige Möglichkeit, ein Doktor-Hopping und daraus resultierende unnötige diagnostische und therapeutische Maßnahmen zu verhindern. Gespräche im Rahmen der psychosomatischen Grundversorgung sowie das Angebot von Entspannungsverfahren sind in einer ambulanten HNO-Versorgung durchführbar. Hypnose sollte nur im Rahmen eines psychotherapeutischen Settings angewendet werden. In vielen Fällen wird keine „Heilung“, sondern nur eine „Betreuung“ der Patienten möglich sein. Es ist zu beachten, dass chronische Schmerzzustände zu Persönlichkeitsveränderungen und Depressionen führen können. Patienten mit Glossodynie sind gegebenenfalls in Zusammenarbeit mit einer Schmerzambulanz oder einem Schmerztherapeuten zu therapieren. Nicht induzierte Therapieversuche sind zu vermeiden, da Misserfolge die Symptomatik oft weiter verstärken.

\subsubsection{Globus pharyngeus}

Die Patienten leiden an einem Kloß- oder Fremdkörpergefühl im Hals, zum Teil auch verbunden mit einer erhöhten Schleimproduktion und Räusperzwang. Der Ausschluss einer malignen Erkrankung steht diagnostisch an erster Stelle. Auch ein gastroösophagealer Reflux sollte bedacht werden. Bei den meisten Patienten wird jedoch keine kausale organ-pathologische Veränderung gefunden. Eine Studie in England fand 5 Tumore bei 699 retrospektiv untersuchten Patienten [79]. Ein psychogenes Globusgefühl ist ein klassisches Beispiel für ein Konversionssyndrom. Konversion im psychodynamischen Sinn bedeutet, dass das Symptom der Preis ist, den der Patient bezahlt, weil er starke negative Gefühle wie Angst, Scham, Trauer und Wut nicht erträgt und nicht wahrhaben will. Das Ich des Patienten drückt die verdrängten Emotionen in Form eines körperlichen Symptoms aus [4]. Das körperliche Symptom neutralisiert den psychischen Konflikt. Mit dem Globus verbunden ist zumeist ein Anspannungszustand der pharyngealen und laryngealen Muskulatur, der sich typischerweise in Stresssituationen verstärkt.

An erster Stelle in der Therapie steht das aufklärende Gespräch nach der sorgfältigen HNO-Untersuchung. Bei erst seit kurzem bestehender Symptomatik ist damit oft schon eine Besserung zu erzielen. Interessant ist, dass bei Patienten mit Konversionsstörungen eine erhöhte Bereitschaft für hypnotische Suggestionen besteht [80]. In einer randomisierten, kontrollierten Studie wurde über ein signifikant im Vergleich zur Wartegruppe besseres Ansprechen auf eine hypnotische Therapie berichtet, welches auch im Follow-up nach sechs Monaten stabil war [81]. Bei chronifizierten Beschwerden konnte auch mit Stimmtherapie eine Verbesserung erzielt werden [82]. Mehrere Einzelfallbeschreibungen und kleinere Studien berichten über Erfolge mit kognitiver Verhaltenstherapie bei Konversionsstörungen sowohl bei Kindern wie auch bei Erwachsenen [83]. In schweren Fällen kommt auch eine zusätzliche Behandlung mit Anxiolytika oder Tranquilizern infrage.

Fazit für die Praxis: Bei diesen Patienten halte ich folgende an verhaltenstherapeutische Instruktionen angelehnte Maßnahmen für sinnvoll: Sorgfältige Untersuchung, aber keine Flucht in aufwendige therapeutische Maßnahmen (die Patienten geben in aller Regel willig ihre Zustimmung auch zu Operationen); Erklä- ren physiologischer und funktioneller Mechanismen im Zusammenhang mit der Konfliktkonstellation; Akzeptieren der Symptomatik und nicht versuchen, dem Patienten das Symptom wegzunehmen; erreichbare Ziele setzen, Belohnen gesunden und Ignorieren krankhaften Verhaltens. Für Hypnosebehandlung und kognitive Verhaltenstherapie ist eine Überweisung zum Fachpsychotherapeuten erforderlich.

\subsubsection{Dysphagie und Phagophobie}

Psychosomatische Aspekte und eine Konversionsstörung spielen oft eine Rolle bei oropharyngealen Schluckstörungen, insbesondere wenn keine organische Ursache gefunden wurde. Betroffen sind meist Frauen mit einem ängstlich-hypochondrischen Verhaltensmuster. Bei emotionalem Stress und psychischer Komorbidität von Angst und Depression tritt eine Dysphagie gehäuft auf [84]. Die Beschwerden treten meist nach einer organisch erklärbaren Schluckstörung wie einer ausgeprägten Tonsillitis auf und persistieren aber nach Abheilen der Primärerkrankung. Sie können sich sogar bis zur Schluckphobie, das bedeutet der Unfähigkeit, feste oder flüssige Nahrung zu sich zu nehmen, steigern $[85,86]$. Die Symptomatik kann auch im Zusammenhang mit einem Globusgefühl auftreten sowie besonders bei Kindern und Jugendlichen in Kombination mit einer Essstörung. Die Unfähigkeit, mit kritischen Lebenssituationen fertig zu werden und zu adaptieren, drückt sich umgangssprachlich in „etwas nicht schlucken können“ oder „es bleibt im Halse stecken“ aus.

Wegen der unterschiedlichen Formen der verhaltenstherapeutischen Ansätze sind Vergleiche schwierig. Kleinere Studien und Einzelfallberichte geben positive Effekte an, größere Gruppenprogramme ergaben keinen Wirksamkeitsnachweis [87]. Verhaltenstherapeutisch orientierte Übungsprogramme mit Änderungen der Ernährung und Schluckübungen sind eine Möglichkeit, diesen Patienten zu helfen $[88,89]$. Phagophobie ist gekennzeichnet durch die Angst vor dem Schlucken und dadurch ausgelöste Schluckbeschwerden. Wenn die Angsterkrankung im Vordergrund steht, ist eine psychologische Diagnostik unumgänglich. Eine kleine Studie mit fünf Schulkindern, die an Globusgefühlen, Dysphagie und Phagophobie litten, beschreibt den erfolgreichen Einsatz von Hypnose und Hypnotherapien im Rahmen verhaltenstherapeutischer Interventionen [90]. Auch für Tumorpatienten, die an psychogen überlagerten Schluckstörungen leiden, kommt dieser therapeutische Ansatz infrage. Bei ausreichenden kognitiven Fähigkeiten des Patienten ist auch ein Videofeedback, welches bereits erfolgreich zur Schluckrehabilitation nach Operationen eingesetzt wurde, zu überlegen [91].

Fazit für die Praxis: Auch von dieser Patientengruppe wird oft zuerst der HNO-Arzt konsultiert. Seine Aufgabe ist der Ausschluss einer behandlungsbedürftigen organischen Erkrankung und die Beratung über weitere Therapieoptionen wie zum Beispiel Schluckübungsprogramme, Videofeedback, Hypnotherapien und kognitive Verhaltenstherapie. Je unbefangener der HNO-Kollege darüber informiert, umso leichter wird es dem Patienten fallen, eine Überweisung zum Fachpsychotherapeuten zu akzeptieren. Für die Gesprächstechnik gilt das beim Globus pharyngeus bereits Gesagte. 


\subsection{Erkrankungen des Larynx}

\subsubsection{Psychogene Dysphonie und psychogene Aphonie}

Patienten mit psychogenen Stimmstörungen konsultieren oft als erstes einen HNO-Arzt oder Phoniater, da sie eine organische Ursache ihrer Beschwerden annehmen. Im weiteren Verlauf wird dann zumeist die Notwendigkeit einer psychotherapeutischen Behandlung ersichtlich. Wichtig ist, dass bei der HNO-ärztlichen Untersuchung die Bereitschaft des Patienten für eine psychotherapeutische Intervention gefördert werden kann. Psychogene Stimmstörungen sind oft verbunden mit Angst, Depression, Konversionssymptomen und Persönlichkeitsstörungen. Sie können psychodynamisch als kreative Fähigkeit eines Menschen gesehen werden, mit einem chronischen inneren Konflikt umzugehen, und die Reaktion auf ein traumatisches Stresserlebnis sein. Oft beginnt die Symptomatik mit Heiserkeit nach einem viralen Infekt [92]. Die hypofunktionelle Form der Aphonie ist häufiger als die hyperfunktionelle. Wenn die Störung noch nicht lange besteht, ist der psychische Konflikt für den Patienten oft noch bewusstseinsnah und damit nachvollziehbar. Eine symptomzentrierte Kurzzeit-Psychotherapie ist dann schon ausreichend. Eine konfliktaufdeckende analytische Therapie wird von vielen Patienten nicht gewünscht [93]. Bei hyperfunktionellen Stimmstörungen kann die Kombination von logopädischer Behandlung mit Biofeedback und kognitiver Verhaltenstherapie erfolgreich sein [94]. Eine kleine neue Pilotstudie kam zu dem Ergebnis, dass wenn Logopädie alleine keine ausreichende Verbesserung erzielt, ein laryngeales und velopharyngeales Biofeedback-Training weiterhelfen kann [95]. Biofeedback ermöglicht den Patienten, die Veränderungen der eigenen Stimme während einer logopädischen Behandlung, die sonst subjektiv schwer zu erfassen sind, besser beurteilen zu können. Bei interpersonellen Konflikten beruflicher oder familiärer Art ergaben sich in einer Studie mit 30 Patienten gute Erfolge mit einer Kombination aus Stimmtherapie und der Förderung kommunikativer Fähigkeiten [96]. Über Hypnotherapie gibt es nur wenige Einzelfallberichte mit zum Teil nur temporären Verbesserungen.

Fazit für die Praxis: Die Abgrenzung zwischen funktionellen und psychogenen Beschwerden ist problematisch, insbesondere, wenn es sich um gemischte Krankheitsbilder mit einer organischen und einer psychischen Komponente handelt [97]. Generell kann man davon ausgehen, dass je kürzer die Beschwerden bestehen, der Patient umso eher mit der Möglichkeit psychischer Auslösefaktoren konfrontiert werden kann. Gerade bei der psychogenen Aphonie kann durch das Provozieren stimmhafter Laute wie Husten oder Räuspern ein Überraschungseffekt und oft auch eine Besserung der Symptomatik erzielt werden. Empathisches Verhalten des Arztes und Angstreduktion sind entscheidende Therapieziele. Entspannungsverfahren wie auch Biofeedback sind daher sinnvoll auch in Kombination mit Logopädie, mit dem Ziel, die oft pathologische Muskelanspannung im Bereich der Larynxregion zu reduzieren. Bei ausgeprägten hypofunktionellen Störungen steht die Stimmtherapie im Vordergrund. Tiefer gehende seelische Konflikte sind nur durch psychotherapeutische Interventionen, verhaltenstherapeutisch oder tiefenpsychologisch fundiert, behandelbar.

\subsubsection{Laryngeale Dysfunktionen: Laryngospasmus und Laryngismus}

Paroxysmal auftretender Laryngospasmus bedeutet ein periodisches Auftreten von Atemnot in Kombination mit einer Stimmstörung, gekennzeichnet durch eine paradoxe, inspiratorische Adduktion der Stimmbänder. Laryngismus oder laryngeale Dystonie ist ein permanent vorhandener Anspannungszustand der laryngealen Muskulatur, der sich klassischerweise auf Injektionen mit Botulinumtoxin A bessert, der Laryngospasmus jedoch nicht. Es handelt sich um Krankheitsbilder mit organisch nachweisbaren Befunden, die allerdings psychisch überlagert sein können. Es wurde bisher kein psychogener Auslösemechanismus nachgewiesen. Depressive Verstimmungen und Angstzustände wurden bei diesen Patienten gehäuft beobachtet, aber als psychische Reaktion auf die Organerkrankung interpretiert, die sich auch nach einer Botulinumtoxinbehandlung besserte [98]. Stimmtherapie in Kombination mit Psychotherapie sowie ausführliche Aufklärung des Patienten und seiner Familie über die Ätiologie der Erkrankung sind sinnvolle therapeutische Maßnahmen, die aber auch nicht immer befriedigende Ergebnisse zeigen $[99,100]$.

Fazit für die Praxis: Bei diesen Krankheitsbildern steht die organisch orientierte Therapie im Vordergrund. Begleitend erscheinen Entspannungsverfahren, Biofeedback und - bei psychischer Komorbidität wie Angst und Depression - psychotherapeutische Interventionen, wie bereits bei der psychogenen Dysphonie genannt, als sinnvoll.

\subsection{Bruxismus und temporo-mandibuläre Dysfunktion}

Beide Erkrankungsmodalitäten können auch als orale Parafunktionen bezeichnet werden, sie haben gemeinsame Aspekte und zumeist eine multifaktorielle Ursache. Die Patienten suchen oft wegen Ohrenschmerzen oder myofaszialen Schmerzen einen HNO-Arzt auf, der keinen pathologischen Befund in diesem Bereich erheben kann. Manualtherapeutisch ausgebildete Kollegen können in Zusammenarbeit mit einem Zahnarzt oder Kieferorthopäden vorhandene Malokklusionen und Asymmetrien im Bereich des Kiefergelenks therapeutisch angehen. Bei therapieresistenten Patienten müssen auch psychosomatische Komponenten bedacht werden. In mehreren neueren kontrollierten Studien zeigte sich ein Zusammenhang von Bruxismus mit psychischen Faktoren wie Depression, Panikstörungen, ängstlicher Erwartungshaltung und besonders bei Frauen einer erhöhten Empfindlichkeit gegen Stressbelastung [101-103]. Unzufriedenheit im Beruf, aber auch Schichtarbeit, führten zu vermehrtem Auftreten von Bruxismus [104].

Infolge dieser Ergebnisse werden, und dies auch seit vielen Jahren, Entspannungsverfahren, Biofeedback und psychotherapeutische Interventionen empfohlen [105]. Bei Kindern und einem erhöhten Angstlevel sind muskelrelaxierende Verfahren eine sinnvolle Therapieoption [106]. Die Mehrzahl der Patienten mit Bruxismus und mit temporo-mandibulärer Dysfunktion spricht positiv auf muskelrelaxierende Therapie an, sei es in Form von Entspannungsverfahren oder mit Hilfe von Biofeedback-Methoden [107]. 
Kognitive Verhaltenstherapie mit dem Erlernen von Coping-Strategien sowie Stress-Management sind weitere Therapieempfehlungen, ebenso der kurzfristige Einsatz von Benzodiazepinen $[108,109]$. Eine Besserung der Symptomatik durch Hypnose ist nur in Einzelfällen beschrieben worden.

Fazit für die Praxis: Die Aufklärung der Patienten über physiologische Vorgänge beim Kauen und Zähneknirschen und die Zusammenarbeit mit einem Zahnarzt steht am Beginn der Behandlung. Dann folgen die verschiedenen Möglichkeiten der Entspannungsverfahren und Stressbearbeitung, wobei die Arbeitsbedingungen des Patienten von Bedeutung sein können. Wenn Patienten es wünschen, kann auch Hypnose als Methode der Tiefenentspannung in Erwägung gezogen werden. Therapieresistente Fälle oder Patienten mit einer psychischen Komorbidität benötigen psychotherapeutische Behandlung auch unter dem Aspekt, dass die Entwicklung eines chronischen Schmerzsyndroms verhindert wird.

\subsection{Dysmorphophobie}

Bei der Zunahme der Nachfrage nach ästhetisch-plastischen Eingriffen auch im HNO-Bereich sind Kenntnisse über das psychiatrische Krankheitsbild der Dysmorphophobie oder körperdysmorphen Störung von Vorteil. Es handelt sich dabei um Patienten, die zwanghaft auf der Suche nach der ewigen Jugend mit ihrer körperlichen Attraktivität („Dorian-Gray-Syndrom“) beschäftigt sind. Sie sind fokussiert auf kleine vorhandene oder eingebildete Mängel ihrer körperlichen Erscheinung [110]. Es besteht eine erhebliche Diskrepanz zwischen dem subjektiven Erleben des Patienten und den objektivierbaren Befunden. Emotionaler Stress und sozialer Rückzug sind oft die Folgen. Die Patienten drängen auf chirurgische Korrekturen, besonders betroffen im HNO-Bereich sind die Nase, die Augenlider, die Ohrmuscheln sowie auch das gesamte Gesicht. Typischerweise sind diese Patienten oft voroperiert, aber niemals damit zufrieden. Depressionen und Angstzustände bis zur sozialen Phobie sind häufige Komorbiditäten.

Fazit für die Praxis: Da es sich um ein psychiatrisches Krankheitsbild handelt ist die Überweisung zum Psychiater sinnvoll, nur meist wird der Patient diesem Rat nicht folgen. Der HNOArzt kann diese Störung nicht behandeln, sie wird auch durch eine plastisch-ästhetische Maßnahme nicht verbessert. Der Chirurg riskiert eher eine Klage, weil der Patient mit dem Ergebnis immer unzufrieden sein wird. Daher ist es wichtig, diese Störung aufgrund der oben genannten Kriterien zu erkennen und gegebenenfalls eine Operation abzulehnen.

\subsection{Maligne Erkrankungen des HNO-Gebietes}

Die Erkrankung „Krebs“ löst in nahezu jedem Menschen existenzielle Ängste aus. Die meisten unserer Patienten verbinden damit Schmerzen, Leiden und Tod. Die Mitteilung der Diagnose führt bei immerhin bis zu einem Drittel der Tumorpatienten zu einem post-traumatischen Stresssyndrom im Sinne eines psychischen Traumas [111]. Zusätzlich zu der organbedingten Störung kommt es im Laufe einer lebensbedrohlichen Erkrankung oft zu einer erheblichen Reduktion der Lebensqualität meist durch Depressionen und Angstzustände. Die bösartigen Tumore des HNO-Bereiches sind noch durch zusätzliche Besonderheiten gekennzeichnet: 1. Sie sind oft sichtbar oder die Behandlung hin- terlässt sichtbare Narben, das bedeutet, die Patienten sind für ihre Umwelt stigmatisiert und 2. in vielen Fällen von Pharynxund Larynxtumoren ist die Kommunikations- und Schluckfähigkeit nach der Behandlung deutlich beeinträchtigt. In Studien zeigen Patienten nach Laryngektomie erhebliche Einschränkungen im Bereich der sozialen Akzeptanz, der sozialen Aktivitäten, der sexuellen Aktivität sowie der Kommunikationsfähigkeit [112]. Zwanzig bis vierzig Prozent der Tumorpatienten erleiden eine erhebliche emotionale Beeinträchtigung. Problematisch ist, dass in einer Studie bei Patienten mit HNO-Tumoren und Patienten mit niedrigem sozialen Status das ärztliche Erkennen der Distressbelastung besonders schlecht und infolgedessen die psychosoziale Unterstützung unzureichend war [113]. Besonders jüngere Patienten erleben eine stärkere Beeinträchtigung der Lebensqualität und mehr Angst als ältere. Sie benötigen daher mehr Unterstützung [114]. Angst und Depressivität sind insbesondere präoperativ stark erhöht, bei Frauen stärker als bei Männern. Eine ungünstige Krankheitsverarbeitung liegt bei defensiven CopingStrategien wie Misstrauen, kognitiver Vermeidung und Gefühlskontrolle vor. Suchtverhalten, bei HNO-Tumor-Patienten besonders ausgeprägt in Bezug auf Alkohol und Nikotin, gehört auch dazu [115].

Die Interventionsmöglichkeiten sind hauptsächlich vom Zeitpunkt abhängig. Für die Patienten steht die organbezogene Behandlung an erster Stelle und sie sind oft erst nach erfolgter Behandlung und Rehabilitation bereit, sich tiefer mit den Ereignissen auseinander zu setzen und psychotherapeutische Hilfe in Anspruch zu nehmen. Bei HNO-Patienten kommt oft noch die Rehabilitation der Schluckfunktion, der Sprechfunktion und der Stimme soweit wie möglich dazu. Verschiedene Therapieformen haben sich zur Intervention bewährt: supportive Einzelberatung und Krisenintervention, alle Formen meditativer oder entspannungsfördernder Maßnahmen, verhaltenstherapeutische Maßnahmen (zentriert auf Entspannung, Erlernen von Coping-Strategien, positives Denken, „reframing“, kognitive Umstrukturierung etc.), ausgedehnte tiefenpsychologisch fundierte Einzelbehandlung sowie Paar- und Gruppentherapien [116]. Nach wie vor wird die Frage, ob durch psychosoziale Interventionsmaßnahmen auch die Überlebenszeit beeinflusst wird, sehr kontrovers diskutiert. Aber eine Verbesserung der emotionalen Situation Betroffener sollte im Fokus des Interesses der Behandlung stehen. Dabei geht es besonders um die Reduktion von Angst, Depression und Verzweiflung, einen Zuwachs des Gefühls an Kontrolle, die Vermittlung von Wissen über die Erkrankung und ihre Behandlungsmöglichkeiten, eine Stärkung des Selbstwertgefühls sowie eine Verbesserung der Compliance und Lebensqualität.

Fazit für die Praxis: Der behandelnde HNO-Arzt ist für den Tumorpatienten der wichtigste Ansprechpartner, er behandelt den Tumor, er sichert das Überleben. Er sollte über das Ausmaß der individuellen psychischen und sozialen Beeinträchtigungen seines Patienten informiert sein, Empathie offen zeigen, erkennen können, ob sein Patient einen besonderen Betreuungsbedarf hat und die weiteren notwendigen Maßnahmen koordinieren [117]. Das jedem Menschen eigene Körperbild ist durch den Tumor und noch verstärkt durch Operation und Bestrahlung erheblich gestört. Des Weiteren ist die Sprechfähigkeit oft beeinträchtigt. Ich halte daher auch körperbetonte Therapieformen, bei denen der Patient nicht reden muss, für geeignet. 
Der Umgang mit Sterben und Tod führt auch den Arzt oft an die Grenzen seiner psychischen Belastbarkeit. Jeder todkranke Patient hat das Recht, über seine Ängste und Nöte zu reden, jeder Arzt die Pflicht, ihm diese Möglichkeit zu gewähren. Dies wird ihm jedoch nur gelingen, wenn er sich selbst mit dieser Thematik beschäftigt hat und er eigene Ängste nicht verdrängen muss oder - noch schlimmer - auf den Patienten überträgt. Selbsterfahrung und Balintgruppenarbeit sollten für onkologisch tätige Ärzte eine Pflicht sein.

\section{4 Übersicht therapeutischer Verfahren}

\subsection{Entspannungsverfahren 4.1.1 Autogenes Training}

Autogenes Training (AT) ist ein Verfahren zur konzentrierten Selbstentspannung, das als Einzel- sowie als Gruppentherapie möglich ist. Mittels aufeinander aufgebauter Übungen erfolgt eine Sensibilisierung für körperliche Vorgänge und deren Beeinflussung.

Es ist ein von den gesetzlichen und privaten Krankenkassen anerkanntes Verfahren und damit im Rahmen eines Zusatzbudgets beziehungsweise über GOÄ abrechenbar.

Voraussetzungen für die Durchführung und Abrechnung sind von den Landesärztekammern anerkannte Fortbildungskurse und ein Antrag an die jeweilige kassenärztliche Vereinigung.

\subsubsection{Progressive Muskelrelaxation}

Entspannung wird bei der Progressiven Muskelrelaxation (PM) durch den Wechsel von Anspannung und Entspannung bestimmter Muskelgruppen erreicht. Das natürliche Entspannungserlebnis, das auf eine starke Anspannung erfolgt, wird genutzt und in Entspannungssuggestionen eingebaut. Das Verfahren kann von Therapeuten, aber auch in Form von Tonbändern oder in der Kombination, genutzt werden.

Die Progressive Muskelrelaxation ist ein von den gesetzlichen und privaten Krankenkassen anerkanntes Verfahren und damit im Rahmen eines Zusatzbudgets abrechenbar. Es gibt eine ganze Reihe von Studien, welche die Wirksamkeit bei psychosomatischen Erkrankungen belegen. Die Voraussetzungen für die Durchführung und Abrechnung sind analog zum Autogenen Training.

\subsubsection{Biofeedback}

Biofeedback bedeutet eine Rückmeldung der Aktivität physiologischer Vorgänge in Form von Signalen optischer, akustischer oder anderer Art, mit dem Ziel, die eigene bewusste Steuerung scheinbar autonomer körperlicher und seelischer Vorgänge zu ermöglichen.

Biofeedback ist ein nur von den privaten Krankenkassen bezahltes Verfahren und wird fast immer im Einzelsetting durchgeführt. Der Nutzen für die klinische Anwendung ist belegt, das Wirkungsspektrum wurde früher jedoch oft überschätzt [118]. Die Voraussetzung für die Durchführung des Verfahrens ist die Anschaffung eines Gerätes. Die Einweisung wird in der Regel von den Firmen übernommen. Eine weitere Ausbildung ist nicht erforderlich.

\subsubsection{Yoga, Qi Gong, Tai Chi}

Yoga ist die wohl älteste, seelenheilkundliche Beeinflussung und umfasst ein System, das durch Seelentechniken und körperliche Praktiken zu höchster Weisheit führen und von Leid befreien will. Die Hauptanwendung ist nach wie vor im nicht-therapeutischen Bereich, allerdings werden zuverlässige und auch langfristige Verbesserungen besonders bei Angst- sowie Spannungszuständen gefunden [118]. Es gibt keine Abrechnungsmöglichkeiten im Rahmen des EBM oder der GOÄ. Die Krankenkassen bezuschussen aber oft entsprechende Kurse an den Volkshochschulen oder in Fitnessstudios.

Als alternatives Heilverfahren erweckt Qi Gong immer mehr Aufmerksamkeit. Es bedeutet frei übersetzt „Energieübung“ und besteht aus harmonischen Bewegungsfolgen, Atemübungen und einer konzentrierten Lenkung der Vorstellungskraft. Die Haupteinsatzbereiche sind die Erhaltung der körperlichen und geistigen Energie sowie, im Falle einer Erkrankung, die Wiedererlangung der Leistungsfähigkeit und Verbesserung der Lebensqualität. Für die Abrechnung und Durchführung gilt das für Yoga bereits Gesagte.

Tai Chi, bei uns auch als „Schattenboxen“ bekannt, umfasst Bewegungsübungen, die Atmung und Bewegung in einem strengen Ritual vereinigen. Es hat eine entspannende Wirkung und fördert die Körperkondition und Koordination.

Für die Abrechnung und Durchführung gilt das für Yoga bereits Gesagte.

\subsection{Hypnose}

Hypnose ist ein ärztliches, psychotherapeutisches Verfahren. Es bedient sich der therapeutischen Nutzung von Trancezuständen und die Aufgabe des Therapeuten besteht darin, problematischen Prozessen oder traumatischen Erlebnissen einen neuen Kontext zu geben. Es ist ein suggestives Verfahren. Eine Trance ist ein natürliches Phänomen, das bei vielen Menschen induziert werden kann. In diesem Zustand können die Ressourcen eines Menschen erweitert und gestärkt werden. Hypnotherapeutische Verfahren können gut mit anderen Entspannungsverfahren wie zum Beispiel Progressiver Muskelrelaxation kombiniert werden.

Die Anwendung setzt eine Weiterbildung an anerkannten Fortbildungsinstituten voraus. Es kann dann bei entsprechender Qualifikation des Arztes sowohl über die gesetzlichen als auch privaten Krankenkassen abgerechnet werden.

\subsection{Gespräche im Rahmen der Psychosomatischen Grundversorgung}

Die „Psychosomatische Grundversorgung“ ist eine Zusatzqualifikation (keine Zusatzbezeichnung), die in von der jeweiligen Landesärztekammer anerkannten Kursen erworben werden kann. Voraussetzung sind mindestens 50 Stunden Weiterbildung, davon 20 Stunden Theorie und 30 Stunden Übungen zur verbalen Intervention. Des Weiteren werden 30 Stunden Balintgruppen benötigt. Es gibt mittlerweile auch private Versicherungen, die bei Abrechnung psychotherapeutischer Ziffern die Qualifikation des Arztes fordern. 


\subsection{Verhaltenstherapie}

Die Verhaltenstherapie geht davon aus, dass das Umgehen mit Erfahrungen im Leben und damit auch mit Erkrankungen durch die erlebten Konsequenzen und ihre Integration in das Selbst erklärbar ist. Durch eine Neubewertung von Situationen und dem Lernen neuer Verhaltensweisen oder angemessener Problemlösestrategien gelingt es, Krankheitssymptome oder ihre Wahrnehmung zu verändern. Körperwahrnehmung, Krankheitsbewältigung und Patientenschulung sind bei Asthma und Neurodermitis bereits wissenschaftlich evaluiert in die Therapie integriert [72].

Zur Durchführung der Verhaltenstherapie wird entweder der Facharzt für Psychotherapie oder die Zusatzbezeichnung Psychotherapie benötigt. Diese Therapieform wird oft auch von ausgebildeten Psychologen durchgeführt. Die Ausbildungsrichtlinien und ermächtigten Institute sind bei den jeweiligen Landesärztekammern zu erfragen.

\subsection{Tiefenpsychologisch fundierte Gesprächstherapie}

Die Tiefenpsychologie und Analyse sehen die Erfahrungen eines Menschen in den ersten Lebensjahren als Schlüssel zum Verständnis seines Verhaltens und Erlebens. Neurotische Störungen wie Depressionen oder auch Angststörungen sind Behandlungsindikationen.

Zur Durchführung dieser Therapie wird entweder der Facharzt für Psychotherapie oder die Zusatzbezeichnung Psychotherapie benötigt. Die Ausbildungsrichtlinien und ermächtigten Institute sind bei den jeweiligen Landesärztekammern zu erfragen.

Eine tiefenpsychologisch fundierte Gesprächstherapie hat ein festes Setting, das bedeutet immer zur gleichen Zeit ein- bis zweimal pro Woche eine Sitzung von 50 Minuten. In der Regel werden mindestens 25 Stunden benötigt, in schwierigen Fällen können es aber auch mehr als hundert sein.

Für den HNO-Arzt zu beachten sind die Ergebnisse einer neueren Studie zum Stand der ambulanten psychotherapeutischen Versorgung in Deutschland [119]. Patienten warten im Durchschnitt 4,6 Monate auf eine Psychotherapie und jeder zweite Patient, der eine Therapie haben möchte, wird abgelehnt. Aufgrund der unterschiedlichen Bezahlung der gesetzlichen und privaten Krankenkassen warten die gesetzlich versicherten Patienten länger als Privatpatienten auf einen Therapieplatz.

\subsection{Psychopharmaka}

Die Gabe von Psychopharmaka kann adjuvant zu psychosomatischen Therapieverfahren sinnvoll sein, insbesondere wenn die Patienten zusätzlich zu ihren körperlichen Beschwerden unter starken Angstgefühlen und/oder Depressionen leiden.

Die Verordnung ist bei akuten, reaktiven Beschwerden auch durch den HNO-Arzt möglich, sollte aber nicht ohne begleitende, stützende Gespräche erfolgen. Eine psychiatrische Konsiliaruntersuchung ist bei allen schwereren psychischen Erkrankungen, bei Therapieresistenz oder Verläufen über drei Monate erforderlich.
Geeignete Medikamente für Angststörungen sind Benzodiazepine (Bromazepam-Lexotanil ${ }^{\circledR}$, Lorazepam-Tavor ${ }^{\circledR}$ ), wobei das Suchtpotenzial zu beachten ist und der Patient über dieses auch aufgeklärt werden muss. Bei Depressionen sind eher Neuroleptika angezeigt, wobei besonders bei Anspannungs- und Unruhezuständen die neueren sedierenden Serotonin-WiederaufnahmeHemmer (SSRI) aufgrund des geringen Nebenwirkungsspektrums zu bevorzugen sind [120]. Ein Suchtpotenzial ist hier zu vernachlässigen.

\section{Stellenwert der stationären psychosomatischen Behandlung}

Die Behandlung eines psychosomatisch kranken Patienten sollte aus folgenden Gründen zunächst ambulant versucht werden: Zum einen sind natürlich die Kosten einer ambulanten Therapie geringer als die einer stationären. Der andere Grund ist, dass der Patient mit sich, seinem sozialen Umfeld und seiner Erkrankung zurecht kommen muss. Wenn er psychisch gesund werden will ist er gezwungen, zu adaptieren, zu habituieren als auch CopingStrategien zu entwickeln. Und dies alles muss auch zu Hause und nicht nur im geschützten Klinikrahmen funktionieren. Dies bedeutet, dass die stationäre Behandlung einer psychosomatischen Erkrankung nur unter folgenden Umständen zu rechtfertigen ist [121]:

- eine ambulante Therapie hat sich als nicht ausreichend erwiesen und gleichzeitig lässt eine stationäre Behandlung einen Therapieerfolg erwarten;

- die psychische Instabilität des Patienten (insbesondere bei begleitenden Angststörungen und Depressionen mit Suizidalität oder bei Persönlichkeitsstörungen mit extrem starkem Leidensdruck) ist so ausgeprägt, dass eine engmaschige Betreuung notwendig ist;

- bei schweren psycho-sozialen Konfliktsituationen (insbesondere Gewalt in der Familie oder Missbrauch), die eine vorübergehende Distanzierung von dem familiären Umfeld notwendig erscheinen lassen.

Ob eine stationäre psychosomatische Behandlung (Krankenhausbehandlung) oder eine stationäre psychosomatische Heiloder Vorsorgebehandlung (früher „Kur“, heute „medizinische Maßnahme zur Rehabilitation“, „Rehabilitationskur“, ,stationäre Vorsorgemaßnahme") notwendig ist, hängt von der Schwere der Erkrankung und der notwendigen Intensität der Betreuung ab.

\section{Prognose psychosomatischer Störungen und Ausblick in die Zukunft}

Die Prognose einer psychosomatischen Erkrankung hängt entscheidend von der Erkrankungsdauer und der Anzahl der bereits erfolgten, meist erfolglosen, Behandlungsversuche ab. Eine psychiatrische Komorbidität wie Angst und Depression geht meist mit einem erhöhten Schweregrad der Störung und einer schlechteren Prognose einher. 
Auf der Patientenseite sind die Ressourcen des Patienten, seine intellektuellen Fähigkeiten und sein soziales Umfeld von entscheidender Bedeutung zur Entwicklung geeigneter CopingStrategien für den Umgang mit seiner Erkrankung.

Auf der Seite des HNO-Arztes ist es die Chance des Organmediziners, dass viele Patienten erstmals mit psychosomatischen Störungen zu ihm kommen. Hierdurch hat er die Möglichkeit, dem Patienten mit einem psychosomatischen Therapieansatz zu helfen und eine Chronifizierung der Beschwerden zu vermeiden.

Außerdem bedeutet eine frühzeitige und erfolgreiche Behandlung eine Vermeidung von Doktor-Hopping und kostenintensiven Patientenkarrieren; ein wichtiger Aspekt bei den knappen Ressourcen unseres Gesundheitssystems.

Als Ärzte sollten wir dabei zudem nicht vergessen, dass auch wir nicht beliebig belastbar sind. Unsere berufliche Situation ist heutzutage angefüllt mit Vorschriften und Zwängen jeglicher
Art. Um ein Burn-Out-Syndrom zu vermeiden brauchen wir Erfolgserlebnisse. Der erfolgreiche Umgang mit diesen oft schwierigen Patienten wirkt sich nicht zuletzt sehr positiv auf unser ärztliches Selbstverständnis aus und vermeidet Frustrationen.

\section{Danksagung}

Frau Prof. Dr. phil. Ellen Aschermann, Psychologisches Institut der Universität zu Köln, hat mir durch ihre Unterstützung und konstruktive Kritik zum Manuskript wertvolle Hilfe geleistet. Zahlreiche Verbesserungsvorschläge von Frau cand. phil. Verena Lissy sind in das Manuskript miteingeflossen. Den genannten und weiteren ungenannten Personen sei herzlich gedankt für die gewährte Hilfe und Durchsicht des Manuskriptes. 


\section{Literatur}

${ }^{1}$ Lieberz K, Heger S, Hofmann HP, Schmölder H (Hrsg). Kompendium der Psychosomatik und Psychotherapeutischen Medizin. Köln: Marek-Verlag, 2002: 10

${ }^{2}$ Bergner T. Lebensaufgabe statt Lebens-Aufgabe. Dtsch Arztebl 2004; 101: A2232-2234

${ }^{3}$ Greimel KV, Biesinger E. Psychologische Prinzipien bei der Behandlung von Tinnituspatienten. HNO 1999; 47: 130-134

${ }^{4}$ von Uexküll T (Hrsg). Psychosomatische Medizin. München: Urban \& Schwarzenberg, 1996

${ }^{5}$ Goebel G, Hiller W. Tinnitus-Fragebogen (TF). Standardinstrument zur Graduierung des Tinnitusschweregrades. Ergebnisse einer Multicenterstudie mit dem Tinnitus-Fragebogen (TF). HNO 1994; 42: $166-172$

${ }^{6}$ Lenarz T. Diagnostik und Therapie des Tinnitus. Laryngo-Rhino-Otol 1998; 77: $54-60$

${ }^{7}$ Zenner HP. Kognitive Tinnitusdesensitivierung- evidenzbasierte und leitliniengerechte Habituationstherapie bei chronischer Tinnitussensitivierung. HNO 2003; 51: 687-689

${ }^{8}$ Schilter B, Jäger B, Heermann R, Lamprecht F. Medikamentöse und psychologische Therapien bei chronischem subjektivem Tinnitus. HNO 2000; 48: 589-597

${ }^{9}$ Hazell JWP. The TRT method in practice. In: Hazell JWP (ed). Proceedings of the Sixth International Tinnitus Seminar. Cambridge UK: Oxford University Press, 1999: 92 - 98

${ }^{10}$ Jastreboff PJ, Jastreboff MM. Tinnitus Retraining Therapy (TRT) as a method for treatment of tinnitus and hyperacusis patients. J Am Acad Audiol 2000; 11: $162-177$

${ }^{11}$ von Wedel H, von Wedel UC. Eine Bestandsaufnahme zur TinnitusRetraining-Therapie. HNO 2000; 48: 887-901

${ }^{12}$ Goebel G, Rübler D, Stepputat F, Hiller W, Heuser J, Fichter MM. Controlled prospective study of tinnitus retraining therapy compared to tinnitus coping therapy and broad-band noise generator therapy. In: Hazell JWP (ed). Proceedings of the Sixth International Tinnitus Seminar. Cambridge UK: Oxford University Press, 1999: 302 - 306

${ }^{13}$ Goebel G, Keeser W, Fichter M, Rief W. Neue Aspekte des komplexen chronischen Tinnitus. Teil I: Überprüfung eines multimodalen verhaltensmedizinischen Behandlungskonzeptes. Psychother Psychosom Med Psychol 1991; 41: 115-122

${ }^{14}$ Härter M, Maurischat C, Weske G, Laszig R, Berger M. Psychische Belastungen und Einschränkungen der Lebensqualität bei Patienten mit Tinnitus. HNO 2004; 52: 125-131

${ }^{15}$ Hiller W, Goebel G. A psychometric study of complaints in chronic tinnitus. J Psychosom Res 1992; 36: 337 - 348

${ }^{16}$ D’Amelio R, Archonti C, Scholz S, Falkai P, Plinkert PK, Delb W. Akuter Tinnitus. Psychische Korrelate und Ausmaß der Belastung bei Patienten. HNO 2004; 52: 599-603

${ }^{17}$ Goebel G, Keeser W, Fichter M, Rief W. Teil II: Die verlorene Stille: Auswirkungen und psychotherapeutische Möglichkeiten beim komplexen chronischen Tinnitus. Psychother Psychosom Med Psychol 1991; 41: $123-133$

${ }^{18}$ Kirsch CA, Blanchard EB, Parnes SM. A multiple-baseline evaluation of the treatment of subjective tinnitus with relaxation training and biofeedback. Biofeedback Self Regul 1987; 12: 295-312

${ }^{19}$ Landis B, Landis E. Is biofeedback effective for chronic tinnitus? An intensive study with seven subjects. Am J Otolaryngol 1992; 13: $349-356$

${ }^{20}$ Podoshin L, Ben-David Y, Fradis M, Gerstel R, Felner H. Idiopathic subjective tinnitus treated by biofeedback, acupuncture and drug therapy. Ear Nose Throat J 1991; 70: 284-289

${ }^{21}$ Scott B, Lindberg P, Lyttkens L, Melin L. Psychological treatment of tinnitus. An experimental group study. Scand Audiol 1985; 14: $223-230$

${ }^{22}$ Andersson G, Strömgren T, Ström L, Lyttkens L. Randomized controlled trial of internet-based cognitive behavior therapy for distress associated with tinnitus. Psychosom Med 2002; 64: 810-816

${ }^{23}$ Kaldo V, Larsen HC, Jakobsson O, Andersson G. Kognitiv beteendeterapi via Internet. Patienter med tinnitus får hjälp hantera sina besvär - enklare och billigare [Cognitive behavior therapy via Internet. Patients with tinnitus are helped to manage their problem- simpler and cheaper]. Läkartidningen 2004; 101: 556 - 560
${ }^{24}$ Schmidt A, Lins U, Wetscher I, Welzl-Müller K, Weichbold V. Counselling vs. Gruppentherapie bei chronischem Tinnitus. Ein retrospektiver Vergleich der Interventionseffizienz. HNO 2004; 52: 242 - 247

${ }^{25}$ Eysel-Gosepath K, Gerhards F, Schicketanz KH, Teichmann K, Benthien M. Aufmerksamkeitslenkung in der Tinnitustherapie. HNO 2004; $52: 431-438$

${ }^{26}$ Ogata Y, Sekitani T, Moriya K, Watanabe K. Biofeedback therapy in the treatment of tinnitus. Auris Nasus Larynx 1993; 20: 95 - 101

27 Attias J, Shemesh Z, Haim S, Gold S, Shoham C, Garaggi D. Comparison between self-hypnosis, masking and attentiveness for alleviation of chronic tinnitus. Audiology 1993; 32: 205 - 212

${ }^{28}$ Attias J, Shemesh Z, Shoham C, Shahar A, Sohmer H. Efficacy of selfhypnosis for tinnitus relief. Scand Audiol 1990; 19: 245 - 249

${ }^{29}$ Lindberg P, Scott B, Melin L, Lyttkens L. Behavioural therapy in the clinical management of tinnitus. Br J Audiol 1988; 22: 265 - 272

${ }^{30}$ Lindberg P, Scott B, Melin L, Lyttkens L. Long-term effects of psychological treatment of tinnitus. Scand Audiol 1987; 16: 167-172

${ }^{31}$ Tai Chi besserte meinen Tinnitus. Tinnitus-Forum 2001; 2: 37

32 Kröner-Herwig B, Hebing G, van Rijn-Kalkmann U, Frenzel A, Schilkowsky G, Esser G. The management of chronic tinnitus - comparison of a cognitive-behavioural group training with yoga. J Psychosom Res 1995; 39: $153-165$

${ }^{33}$ Curotto Costa D. Tinnitus and hypnosis. Preliminary report. Prensa Med Argent 1961; 48: 444-446

${ }^{34}$ Kaye JM, Marlowe FI, Ramchandani D, Berman S, Schindler B, Loscalzo G. Hypnosis as an aid for tinnitus patients. Ear Nose Throat J 1994; 73 : 309-312

${ }^{35}$ Navratil L. Hypnoseheilung einer psychogenen Gleichgewichtsstörung bei kompletter Ertaubung. Wiener Archiv für Psychologie, Psychiatrie und Neurologie 1953; 3: $211-214$

${ }^{36}$ Steinriede R. Medizinische Hypnose bei Tinnitus und Hörsturz. Heidelberg: Carl-Auer-Systeme-Verlag, 2002

${ }^{37}$ Marks NJ, Karl H, Onisiphorou C. A controlled trial of hypnotherapy in tinnitus. Clin Otolaryngol 1985; 10: $43-46$

${ }^{38}$ Schaaf H, Klofat B, Hesse G. Hyperakusis, Phonophobie und Recruitment. Mit Geräuschempfindlichkeit assoziierte Hörabweichungen. HNO 2003; 51: 1005 - 1011

${ }^{39}$ Nelting M, Hesse G, Schaaf H. Tinnitustherapie mit Leib und Seele. München: Profil, 1998

${ }^{40}$ Schneider WR, Hilk A, Franzen U. Soziale Unterstützung, Beschwerdedruck, Stressverarbeitung und Persönlichkeitsmerkmale bei Patienten mit subjektivem chronischem Tinnitus aurium und einer klinischen Kontrollgruppe. HNO 1994; 42: 22 -27

${ }^{41}$ Hesse G, Rienhoff NK, Nelting M, Laubert A. Ergebnisse stationärer Therapie bei Patienten mit chronisch komplexem Tinnitus. LaryngoRhino-Otol 2001; 80: $503-508$

${ }^{42}$ Hesse G. Retraining und Tinnitustherapie. Stuttgart, New York: Thieme Verlag, 2000

${ }^{43}$ Hoffmeister K. Verhaltensmedizinische Untersuchungen zum Hörsturz. Frankfurt: Peter Lang, 1988

${ }^{44}$ Lamparter U. Psychosomatische Aspekte beim Hörsturz. Versicherungsmedizin 1998; 50: $104-109$

${ }^{45}$ Lamparter U, Schmidt HU. Psychosomatic medicine and otorhinolaryngology. Psychother Psychosom 1994; 61: 25 - 40

${ }^{46}$ Dohse J, Lehrl S, Berg M. Personality system and sudden deafness: a comparative psychological study. Adv Otorhinolaryngol 1981; 27: $110-113$

${ }^{47}$ Schüssler G, Geishauser E, Rüger U. Psychosomatische Faktoren beim idiopathischen Hörsturz. HNO 1992; 40: 4-9

${ }^{48}$ Kothe C, Fleischer S, Breitfuß A, Hess M. Diagnostik von psychogenen Hörstörungen im Kindesalter. HNO 2003; 51: 915 - 920

${ }^{49}$ Hülse M, Meyer JG. Psychogene Taubheit, ein differenzialdiagnostisches Problem des Hörsturzes. Laryngol Rhinol Otol 1981; 60: 53 - 55

50 Zorowka PG. Psychogene Hörstörungen im Kindes- und Jugendalter. HNO 1992; 40: 386-391

51 Veniar FA, Salston RS. An approach to the treatment of pseudohypacusis in children. Am J Dis Child 1983; 137: 34-36

52 Erlandsson SI, Eriksson-Mangold M, Wiberg A. Menières disease: trauma, distress and adaptation studied through focus interview analyses. Scand Audiol Suppl 1996; 43: 45-56

${ }^{53}$ Schaaf H. Die Menièresche Krankheit. Krieg im Innenohr. Berlin, Heidelberg: Springer, 1995

${ }^{54}$ Eckhardt-Henn A. Psychogener Schwindel legt Patienten länger lahm. Welche seelischen Erkrankungen hinter Schwindel stecken können. MMW Fortschr Med 2000; 142: 30 - 32 
55 Smith MS. Evaluation and management of psychosomatic symptoms in adolescence. Clin Pediatr 1986; 25: 131 - 135

${ }^{56}$ Jacob RG, Furman JM. Psychiatric consequences of vestibular dysfunction. Curr Opin Neurol 2001; 14: 41 - 46

${ }^{57}$ Godemann F, Linden M, Neu P, Heipp E, Dörr P. A prospective study on the course of anxiety after vestibular neuronitis. J Psychosom Res 2004; 56: $351-354$

${ }^{58}$ Fried MP. The evaluation of dizziness in children. Laryngoscope 1980; 90: $1548-1560$

${ }^{59}$ Johansson M, Akerlund D, Larsen HC, Andersson G. Randomized controlled trial of vestibular rehabilitation combined with cognitive-behavioral therapy for dizziness in older people. Otolaryngol Head Neck Surg 2001; 125: $151-156$

${ }^{60}$ Eckhardt A, Tettenborn B, Krauthauser H, Thomalske C, Hartmann O, Hoffmann SO, Hopf HC. Schwindel- und Angsterkrankungen - Ergebnisse einer interdisziplinären Untersuchung. Laryngo-Rhino-Otol 1996; 75: $517-522$

${ }^{61}$ Monzani D, Casolari L, Guidetti G, Rigatelli M. Psychological distress and disability in patients with vertigo. J Psychosom Res 2001; 50: 319-323

62 Eckhardt-Henn A. In: Maurer J (Hrsg). Neurootologie. Stuttgart, New York: G. Thieme Verlag, 1999

${ }^{63}$ Drummond PD, Hewson-Bower B. Increased psychosocial stress and decreased mucosal immunity in children with recurrent upper respiratory tract infections. J Psychosom Res 1997; 43: 271 - 278

${ }^{64}$ Reid MR, Mackinnon LT, Drummond PD. The effects of stress management on symptoms of upper respiratory tract infection, secretory immunoglobulin A, and mood in young adults. J Psychosom Res 2001; 51: $721-728$

${ }^{65}$ Hewson-Bower B, Drummond PD. Secretory immunoglobulin A increases during relaxation in children with and without recurrent upper respiratory tract infections. J Dev Behav Pediatr 1996; 17: $311-316$

${ }^{66}$ Black S. Shift in dose-response curve of Prausnitz-Kustner reaction by direct suggestion under hypnosis. Br Med J 1963b; 1: 990-992

${ }^{67}$ Reimann HJ, Meyer HJ, Wendt P. Stress and Histamine. In: Ring J, Burg G (eds). New trends in allergy. Berlin, Heidelberg, New York: Springer, 1981

${ }^{68}$ Kovács M, Stauder A, Szedmák S. Severity of allergic complaints: the importance of depressed mood. J Psychosom Res 2003; 54: 549-557

${ }^{69}$ Stauder A, Kovács M. Anxiety symptoms in allergic patients: identification and risk factors. Psychosom Med 2003; 65: 816-823

${ }^{70}$ Richter R, Ahrens ST. Psychosomatische Aspekte der Allergie. In: Fuchs E, Schulz KH (Hrsg). Manuale Allergologicum. Deisenhofen: Dustri-Verlag, 2004

${ }^{71}$ Gieler U. Psyche und Allergie. In: Heppt W, Renz H, Röcken M (Hrsg). Allergologie. Berlin, Heidelberg, New York: Springer, 1998

72 Petermann F. Asthma und Allergie. Göttingen: Hogrefe Verlag, 1997

${ }^{73}$ Sullivan PD. The diagnosis and treatment of psychogenic glossodynia. Ear Nose Throat J 1989; 68: 795-798

${ }^{74}$ Rhodus NL, Fricton J, Carlson P, Messner R. Oral symptoms associated with fibromyalgia syndrome. J Rheumatol 2003; 30: 1841 - 1845

${ }^{75}$ Reiss M, Reiss G. Burning mouth syndrome - etiology, differentialdiagnostical aspects and therapy. Ther Umsch 2004; 61: 308 - 312

${ }^{76}$ Miyaoka H, Kamijima K, Katayama Y, Ebihara T, Nagai T. A psychiatric appraisal of „glossodynia“. Psychosomatics 1996; 37: 346-348

${ }^{77}$ Niemeier V, Kupfer J, Brosig B, Gieler U. Glossodynia as an expression of a somatoform disorder. A hard-to-grasp psychodiagnostic disease? With presentation of a single case from a psychodynamic point of view. Dermatol Psychosom 2001; 2: 134-141

${ }^{78}$ Shenefelt PD. Biofeedback, cognitive-behavioral methods, and hypnosis in dermatology: is it all in your mind? Dermatol Ther 2003; 16: $114-122$

${ }^{79}$ Harar RP, Kumar S, Saeed MA, Gatland DJ. Management of globus pharyngeus: review of 699 cases. J Laryngol Otol 2004; 118: 522 - 527

${ }^{80}$ Roelofs K, Hoogduin KA, Keijsers GP, Naring GW, Moene FC, Sandijck P. Hypnotic susceptibility in patients with conversion disorder. J Abnorm Psychol 2002; 111: 390-395

${ }^{81}$ Moene FC, Spinhoven P, Hoogduin KAL, van Dyck R. A randomized controlled clinical trial of a hypnosis-based treatment for patients with conversion disorder. Int J Clin Exp Hyp 2003; 51: 29-50

82 Khalil HS, Bridger MW, Hilton-Pierce M, Vincent J. The use of speech therapy in the treatment of globus pharyngeus patients. A randomised controlled trial. Rev Laryngol Otol Rhinol (Bord) 2003; 124: $187-190$
${ }^{83}$ Donohue B, Thevenin DM, Runyon MK. Behavioral treatment of conversion disorder in adolescence. A case example of Globus Hystericus. Behavior Modification 1997; 21: 231 - 251

${ }^{84}$ Bretan O, Henry MA, Kerr-Correa F. Dysphagia and emotional distress. Arq Gastroenterol 1996; 33: 60-65

85 Berghaus A. HNO-Erkrankungen. In: Deter HC (Hrsg). Angewandte Psychosomatik. Stuttgart: Georg Thieme Verlag, 1997: 385-406

${ }^{86}$ Shapiro J, Franko DL, Gagne A. Phagophobia: a form of psychogenic dysphagia. A new entity. Ann Otol Rhinol Laryngol 1997; 106: $286-290$

${ }^{87}$ Langmore SE. Efficacy of behavioral treatment for oropharyngeal dysphagia. Dysphagia 1995; 10: 259-262

88 Logemann JA. Behavioral management for oropharyngeal dysphagia. Folia Phoniatr Logop 1999; 51: 199-212

${ }^{89}$ Rosenbek JC. Efficacy in dysphagia. Dysphagia 1995; 10: 263 - 267

${ }^{90}$ Culbert TP, Kajander RL, Kohen DP, Reaney JB. Hypnobehavioral approaches for school-age children with dysphagia and food aversion: a case series. J Dev Behav Pediatr 1996; 17: 335-341

${ }^{91}$ Denk DM, Kaider A. Videoendoscopic biofeedback: a simple method to improve the efficacy of swallowing rehabilitation of patients after head and neck surgery. ORL J Otorhinolaryngol Relat Spec 1997; 59: $100-105$

92 Baker J. Psychogenic voice disorders and traumatic stress experience: a discussion paper with two case reports. J Voice 2003; 17: 308 - 318

${ }^{93}$ Mans EJ. Psychotherapeutische Behandlung von Patienten mit funktionellen Stimmstörungen. Folia Phoniatr Logop 1994; 46: 1-8

${ }^{94}$ Sime WE, Healey EC. An interdisciplinary approach to the treatment of a hyperfunctional voice disorder. Biofeedback Self Regul 1993; 18: $281-287$

95 van Lierde KM, Claeys S, de Bodt M, van Cauwenberge P. Outcome of laryngeal and velopharyngeal biofeedback treatment in children and young adults: a pilot study. J Voice 2004; 18: 97-106

96 Andersson K, Schalen L. Etiology and treatment of psychogenic voice disorder: results of a follow-up study of thirty patients. J Voice 1998; 12: $96-106$

${ }^{97}$ Behrendt S, Hess M. Psychotherapeutische Weiterbetreuung stimmgestörter Patienten. HNO 2004; 52: 642 - 647

${ }^{98}$ Kiese-Himmel C, Reeh M, Liebeck H, Zwirner P. Do psychological factors change in patients with spasmodic dysphonia after injections with botulinum-toxin? Laryngorhinootologie 2002; 81: 683-689

${ }^{99}$ Gallivan GJ, Hoffman L, Gallivan KH. Episodic paroxysmal laryngospasm: voice and pulmonary function assessment and management. J Voice 1996; 10: 93 - 105

100 Schmidt M. Nicht alles was pfeift ist Asthma: Zum funktionellen Laryngospasmus. Pneumologie 1993; 47: 439-442

101 Ahlberg J, Savolainen A, Rantala M, Lindholm H, Kononen M. Reported bruxism and biopsychosocial symptoms: a longitudinal study. Community Dent Oral Epidemiol 2004; 32: 307-311

102 Manfredini D, Landi N, Romagnoli M, Bosco M. Psychic and occlusal factors in bruxers. Aust Dent J 2004; 49: 84-89

103 van Selms MK, Lobbezoo F, Wicks DJ, Hamburger HL, Naeije M. Craniomandibular pain, oral parafunctions, and psychological stress in a longitudinal case study. J Oral Rehabil 2004; 31: 738-745

104 Ahlberg K, Ahlberg J, Kononen M, Partinen M, Lindholm H, Savolainen A. Reported bruxism and stress experience in media personnel with and without irregular shift work. Acta Odontol Scand 2003; 61: $315-318$

105 Biondi M, Picardi A. Temporomandibular joint pain-dysfunction syndrome and bruxism: etiopathogenesis and treatment from a psychosomatic integrative viewpoint. Psychother Psychosom 1993; 59: $84-98$

${ }^{106}$ Restrepo CC, Alvarez E, Jaramillo C, Velez C, Valencia I. Effects of psychological techniques on bruxism in children with primary teeth. J Oral Rehabil 2001; 28: 354-360

${ }^{107}$ Foster PS. Use of the Calmset 3 biofeedback/relaxation system in the assessment and treatment of chronic nocturnal bruxism. Appl Psychophysiol Biofeedback 2004; 29: 141 - 147

108 Kato T, Thie NM, Montplaisir JY, Lavigne GJ. Bruxism and orofacial movements during sleep. Dent Clin North Am 2001; 45: 657-684

109 van der Meulen MJ, Lobbezoo F, Naeije M. Role of the psychologist in the treatment of bruxism. Ned Tijdschr Tandheelkd 2000; 107: 297-300

110 Driesch G, Burgmer M, Heuft G. Body dysmorphic disorder. Epidemiology, clinical symptoms, classification and differential treatment indications: an overview. Nervenarzt 2004; 75: 917 -929 
111 Simonton S. Psychological aspects of mind-body medicine: promises and pitfalls from research with cancer patients. Altern Ther Health Med 1998; 4: 50-67

112 Deshmane VH, Parikh HK, Pinni S, Parikh DM, Rao RS. Laryngectomy: a quality of life assessment. Indian J Cancer 1995; 32: 121-130

113 Sollner W, DeVries A, Steixner E, Lukas P, Sprinzl G, Rumpold G, Maislinger S. How successful are oncologists in identifying patient distress, perceived social support, and need for psychosocial counselling? Br J Cancer 2001; 84: 179-185

114 Lee-Preston V, Steen IN, Dear A, Kelly CG, Welch AR, Meikle D, Stafford FW, Wilson JA. Optimizing the assessment of quality of life after laryngeal cancer treatment. J Laryngol Otol 2004; 118: $432-438$

115 Kollbrunner J, Zbaren P, Quack K. Lebensqualitätsbelastung von Patienten mit großen Tumoren der Mundhöhle Teil 2: Krankheitsverarbeitung: Coping, Angst und Depressivität. HNO 2001; 49: 998 - 1007
116 Tschuschke V. Psychologisch-psychotherapeutische Interventionen bei onkologischen Erkrankungen. Onkologe 2003; 9: 657-665

117 Schultz-Coulon HJ. Rehabilitationskonzept für Kehlkopflose. HNO 1984; 32: 3-12

118 Häcker H, Stapf KH (Hrsg). Dorsch Psychologisches Wörterbuch. Bern, Göttingen, Toronto, Seattle: Huber, 1998

${ }^{119}$ Zepf S, Mengele U, Hartmann S. Zum Stand der ambulanten psychotherapeutischen Versorgung der Erwachsenen in der Bundesrepublik Deutschland. Psychother Psychosom Med Psychol 2003; 53: $152-162$

120 Jokinen K, Koskinen T, Selonen R. Flupenthixol versus diazepam in the treatment of psychosomatic disorders: a double-blind, multi-centre trial in general practice. Pharmatherapeutica 1984; 3: 573-581

${ }^{121}$ Goebel G, Decot E, Marek A. Entscheidungshilfen bei Diagnostik und Wahl psychologischer Behandlungsmethoden. HNO 2001; 49: $1036-1047$ 